Elsevier required licence: (C) $<2020>$. This manuscript version is made available under the CC-BY-NCND 4.0 license http://creativecommons.org/licenses/by-nc-nd/4.0/

The definitive publisher version is available online at

[https://www.sciencedirect.com/science/article/pii/S1342937X20302458?via\%3Dihub] 
Research

Elsevier Editorial System(tm) for Gondwana

Manuscript Draft

Manuscript Number: GR-D-20-00212R2

Title: Pathways and challenges of the application of artificial

intelligence to geohazards modelling

Article Type: SI:SPECIAL ISSUE: GR-100

Corresponding Author: Professor Biswajeet Pradhan, PhD

Corresponding Author's Institution: University of Technology Sydney

First Author: Abhirup Dikshit, MSc.

Order of Authors: Abhirup Dikshit, MSc.; Biswajeet Pradhan, PhD; Abdullah Alamri, PhD

Abstract: The application of artificial intelligence (AI) and machine learning in geohazard modelling has been rapidly growing in recent years, a trend that is observed in several research and application areas thanks to recent advances in AI. As a result, the increasing dependence on data driven studies has made its practical applications towards geohazards (landslides, debris flows, earthquakes, droughts, floods, glacier studies) an interesting prospect. These aforementioned geohazards were responsible for roughly $80 \%$ of the economic loss in the past two decades caused by all natural hazards. The present study analyses the various domains of geohazards which have benefited from classical machine learning approaches and highlights the future course of direction in this field. The emergence of deep learning has fulfilled several gaps in: i) classification; ii) seasonal forecasting as well as forecasting at longer lead times; iii) temporal based change detection. Apart from the usual challenges of dataset availability, climate change and anthropogenic activities, this review paper emphasizes that the future studies should focus on consecutive events along with integration of physical models. The recent catastrophe in Japan and Australia makes a compelling argument to focus towards consecutive events. The availability of higher temporal resolution and multi-hazard dataset will prove to be essential, but the key would be to integrate it with physical models which would improve our understanding of the mechanism involved both in single and consecutive hazard scenario. Geohazards would eventually be a data problem, like geosciences, and therefore it is essential to develop models that would be capable of handling large voluminous data. The future works should also revolve towards interpretable models with the hope of providing a reasonable explanation of the results, thereby achieving the ultimate goal of Explainable AI.

Response to Reviewers: Response to Reviewer Comments

Comment: This paper presents an useful overview on the application of machine learning in geohazard modelling in recent years. However, the information is not well presented, the review is too superficial and there are no worthy results. In its present form, the paper needs lots of work. The technical material and the presentation, as it stands in the manuscript, do not merit acceptance for publication. 
Response: The authors are thankful to the reviewer for providing comments and suggestions. As the topic is very broad, we would like to provide an overview of how the review was conducted and what are the takeaways from the article.

The aim of the article was to understand how artificial intelligence (machine learning and deep learning) models have helped to improve our understanding towards geohazards. In this respect, we analysed the use of machine learning models in four different key areas: regression, change detection, prediction and classification. These areas were based on the published article in Nature by Reichstein et al. (2019) which conducted a review of data driven models for geoscience field. We then look into the specific components of geohazards which has benefitted from machine learning models and highlight important review articles where an in-depth information on specific components are provided. Further, we highlight some of the new improvements with the use of deep learning models and suggest future course of action. Figure 4 and Figure 5 illustrates the study flowchart.

The main suggestion from the study are: i) integration of physical model with machine learning models; ii) need to study consecutive events and iii) to test deep learning models with a quest towards "Explainable AI". We have made further changes in the article specifically in section 2 which discusses "Progress in Machine Learning Approaches" to aptly highlight the latest findings and its implications on our understanding.. 


\section{Highlights}

- Analysed machine learning approaches used to understand various geohazard aspects.

- Deep learning models and its improvement over traditional methods was studied.

- Future studies should emphasize on modelling consecutive events using hybrid models.

- We also argue the need for coupling physical process models to data-driven models. 


\section{Geohazard Modelling using AI} techniques

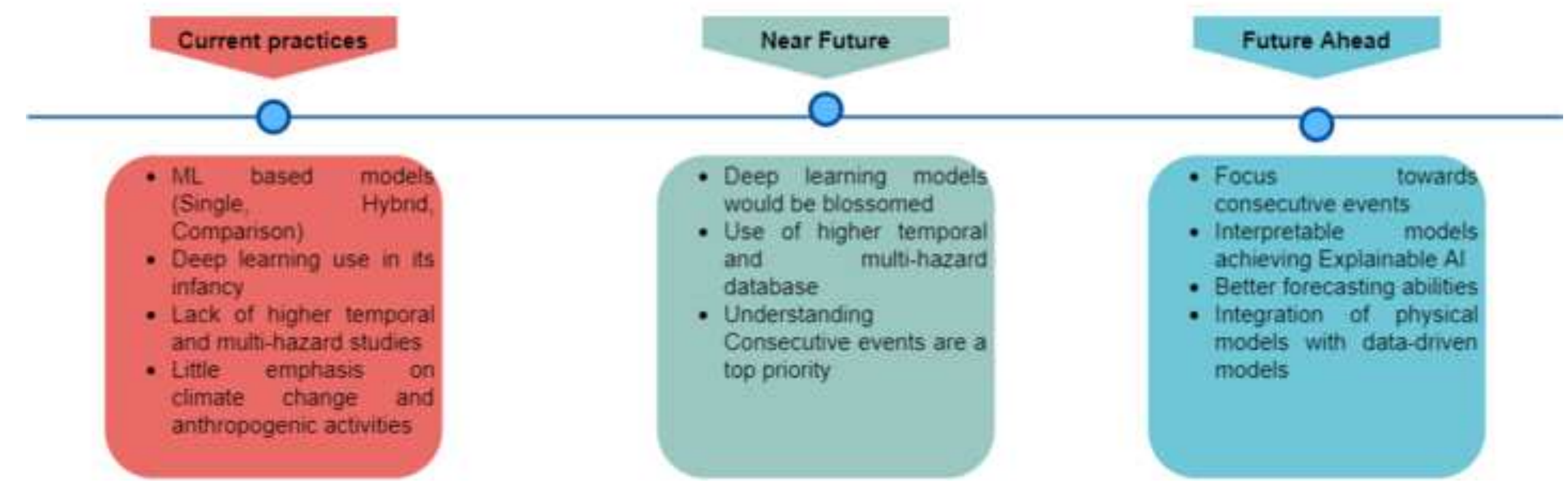


GR Focus Review

\title{
Pathways and challenges of the application of artificial intelligence to geohazards modelling
}

\author{
Abhirup Dikshit ${ }^{1}$, Biswajeet Pradhan ${ }^{1,2,3^{*}}$, Abdullah M. Alamri4 \\ ${ }^{1}$ Centre for Advanced Modelling and Geospatial Information Systems (CAMGIS), Faculty of \\ Engineering \& IT, University of Technology Sydney, Sydney, NSW 2007, Australia \\ ${ }^{2}$ Department of Energy and Mineral Resources Engineering, Sejong University, Choongmu- \\ gwan, 209, Neungdongro Gwangjin-gu, Seoul 05006, Korea \\ 3Center of Excellence for Climate Change Research, Department of Meteorology, King \\ Abdulaziz University, Jeddah 21589, Saudi Arabia \\ 4Department of Geology \& Geophysics, College of Science, King Saud University, Riyadh \\ 11451, Saudi Arabia
}

Corresponding Author: Biswajeet Pradhan, biswajeet.pradhan@uts.edu.au

\begin{abstract}
The application of artificial intelligence (AI) and machine learning in geohazard modelling has been rapidly growing in recent years, a trend that is observed in several research and application areas thanks to recent advances in AI. As a result, the increasing dependence on data driven studies has made its practical applications towards geohazards (landslides, debris flows, earthquakes, droughts, floods, glacier studies) an interesting prospect. These aforementioned geohazards were responsible for roughly $80 \%$ of the economic loss in the past two decades caused by all natural hazards. The present study analyses the various domains of geohazards which have benefited from classical machine learning approaches and highlights the future course of direction in this field. There are several aspects that have made tremendous progress with the overwhelming use of hybrid models, and the availability of large amounts of climatic and remote sensing data. The emergence of deep learning has fulfilled several gaps_in:- especially in improving thei) classification; ii) and seasonal forecasting as well as forecasting at longer lead times; iii) temporal based change detection predictive capability. Apart from the usual challenges of dataset availability, climate change and anthropogenic activities, this review paper emphasizes that the future studies should focus on consecutive events along with integration of physical models. The recent catastrophe in Japan and Australia makes a compelling argument to focus towards consecutive events. The availability of higher temporal resolution and multi-hazard dataset
\end{abstract}


will prove to be essential, but the key would be to integrate it with physical models which would improve our understanding of the mechanism involved both in single hazard-and consecutive hazard scenario. We believe-Ggeohazards would eventually be a data problem, like geosciences, and therefore it is essential to develop models that would be capable of handling large voluminous data. The future works should also revolve towards interpretable models with the hope of providing a reasonable explanation of the results, thereby achieving the ultimate goal of Explainable AI.

Keywords: Machine learning; Deep learning; geo hazards; physical models; Explainable AI

\section{Introduction}

Artificial Intelligence (AI) is a key driver towards a fourth industrial revolution capable of boasting tremendous trans-disciplinary transformations. AI has made giant strides in various applications, from speech recognition (Hinton et al. 2012), self-driving vehicles (Farabet et al. 2012), computer vision (Krizhevsky et al. 2012) and natural language processing (Collobert et al. 2011), which was perceived as a task that could be performed only by humans (Hosny et al. 2018). Although, AI is very broad and there are several applications, in this paper, our discussion will be limited to geohazard studies related to "Machine Learning" and "Deep Learning", which are often used interchangeably with AI and could led the differences among these to be unclear. Although, these terms are related to each other, but they are not same (Fig. 1). Machine learning is a technique of syntactic analysis of data, thereby learning from the data and finally make an informed decision (Alpaydin, 2014). On the other hand, deep learning is a subset of machine learning which is based on neural network structure and is considered being inspired by human brain (LeCun et al. 2015). For deep learning, the model extracts features itself whereas features are manually engineered in the case of classical machine learning (LeCun et al. 2015). While conducting the review, there seems to be quite an overlap of techniques which are almost inevitably described as machine learning, but are not actually machine learning. For e.g. - a lot of data mining approaches have been used and are usually referred as ML, but a key difference between these two exists. Data mining focusses on determining unknown properties in the data without the need of a specific goal, whereas ML needs a goal to perform a task based on known properties gathered from the training data (Buczak and Guven, 2016).

Although, past AI based techniques have led to subhuman performance results, the emergence of recent advanced deep learning algorithms have the capability to outperform human intelligence for specific problems (Mnih et al. 2015; Moravcík et al. 2017). The outperformance of deep learning methods in the game of Go is an accomplishment in itself, long considered being decades away from achieving such a feat given the complex nature of the 
board game involving an enormous number of potential moves (Silver et al. 2016). language translation, writing books, transportation within the next decade (Grace et al. 2017).

Geohazards relate to geological, geomorphological, environmental or anthropogenic activities that have the potential to cause damage to human life, property and/or environment. The geohazard field itself is vast considering the development for different types, it is beyond the scope of this review, and therefore we focus on the six most disastrous geohazards, which are earthquakes, floods, droughts, landslides, debris flows and glacial lake outburst.

The use of AI in the field of geohazards has immense potential in several applications like prediction, change detection (Mezaal et al. 2018), early warning systems (Yang et al. 2019), vulnerability and risk management (Tehrany et al. 2015), spatial modelling (Rahmati et al. 2020) and mitigation measurement (Wang et al. 2019). The availability of vast amounts of remote sensing, climatic and atmospheric data has prompted researchers to identify new ways and techniques of making future reliable geohazards decision (Reichstein et al. 2019). Based on Scopus search, the use of highly advanced and complex algorithms is quite limited in this field when compared to computer science fields like image processing and natural language processing. The present article aims to analyse the current practices and suggest pathways in which the future research direction should proceed.

It is noteworthy to understand that still, most of the works analyse geohazard applications using machine learning techniques which are now slowly moving towards deep learning with few addressing intelligence quotient. In the subsequent sections, we highlight how the development of machine learning algorithms has improved our understanding in geohazard context, and highlight the use of deep learning for the major geohazards.

\section{Progress in Machine Learning Approaches}

The ML algorithms have the capability to learn the underlying system behaviour from a set of training data without the need of prior knowledge existing among the dataset (Schmidt et al. 2019). ML can be divided into three groups, supervised learning, unsupervised learning, and reinforcement learning (Jordan and Mitchell, 2015). Supervised learning attempts to determine an unknown function which correlates the input variables with an output variable, like classification and regression. Whereas, unsupervised learning only has input variable, and the model tries to discover patterns and structures, e.g. -clustering and association (Bishop, 2006). In between them is semi-supervised learning, where a large amount of input data is available but with only few output variables (Nguyen et al. 2017). Finally, 
reinforcement learning treats the problem of finding optimal or sufficiently good actions for a situation (Sutton and Barto, 2018). LeCun et al. (2015) in their path-breaking article on review of deep learning highlighted that unsupervised learning would eventually become more significant as human learning is largely unsupervised.

\subsection{ML in geohazard analysis}

The use of ML for various aspects of geohazard analysis is now pretty popular among researchers. This meteoritic rise in using ML techniques is primarily due to the availability of a vast amount of ground-based, climatic and remote sensing dataset (space-borne, airborne, terrestrial, etc.), along with the increase in computing ability over the past decade. It can be touted as an effective empirical approach for classification (supervised and unsupervised) and/or regression of non-linear systems (Lary, 2016). In the present study, we look into the application of ML in geohazard studies into four key areas: regression, classification, change anømalydetection and prediction.

The underlying assumption in almost every geohazard regression based study is that conditions responsible for occurrences would be the same in the future. Over the past decade, the use of regression techniques has focussed on spatial prediction, i.e., geohazard properties which are comparatively static during the analysed time period. These include geohazard studies related to aspects like susceptibility, vulnerability and risk. Here lies in the main challenge as some of the conditioning factors are inherently dynamic in nature and not static; like effects of climate change and effect of anthropogenic activities. Lately, these dynamics are being considered and regression techniques could help researchers to study such dynamics by analysing the temporal varying parameters. - The classical problem faced by geohazard researchers is classification, of which ML models have gained enough traction and different geohazard aspects have been explored. Like, classifying a region into drought or non-drought areas or classifying into various degrees of susceptibility, hazard, vulnerability, and risk depending on the type of geohazard study being conducted. Further, the use of change anomaly detection techniques is key in almost every geohazard as it helps at two fronts, first being the identification of the historical hazard occurrences which could help in better understanding of the area (like, compiling landslide inventory and glacier outbursts) and second could aid in supporting the mitigation activities immediately (earthquake); or during (drought) the event. Both these aspects are crucial to understand the history of geohazards and providing relief works. But, arguably, the success story in any geohazard study is linked to how good can be the prediction capability, and whether we can predict it with greater accuracy and precision. What makes the field of geohazards so interesting and challenging at the same time as it not only depends on spatial and temporal context like in geosciences and earth sciences, but also on other aspects like the movement 
and volume estimation in case of landslides, glacier outbursts, or the propagation in case of droughts and floods. The further sections will look into which areas of various geohazards have benefitted the most out of ML and its implications on our understanding.

\subsubsection{Landslides and Debris Flows}

The most prominent use of ML for landslide study has been towards regression problems, especially understanding the susceptibility of an affected region. Susceptibility is defined as the likelihood of "where" a geohazard in an area can occur depending on local terrain conditions (Reichenbach et al. 2018; Dikshit et al. 2020a). These methods are primarily based on the analysis of geo-environmental variables which has caused such events historically, either using bivariate or multivariate statistics. The introduction of neural network showed better promising results with respect to logistic regression (Nefeslioglu et al. 2008), and thereafter a quest began to determine the best algorithm for a given study region. The studies have been based either on the use of a single approach like Support Vector Machine (SVM), Random Forest (RF), Artificial Neural Networks (ANN), Logistic Regression (Pradhan et al. 2010; Xu et al. 2012; Yariyan et al. 2020) or have used multiple ML models to find the best technique for a definite study region (Tien Bui et al. 2016; Chen et al. 2017). Landslide susceptibility which has seen the use of several models and found LR, ANN and RF to be most used (Felicísimo et al., 2013; Tien Bui et al. 2016; Chen et al. 2017). Huang and Zhao, (2018) conducted a review on the use of SVM models for landslide susceptibility purposes and suggested that the future studies should involve the use of hybrid models. Similar, has been the case with debris flow susceptibility, e.g.- Xiong et al. (2020) compared four different ML models (LR, SVM, RF and Boosted Regression Trees (BRT)) for Sichuan province in China and found BRT to be performing better. The reclassification of maps generated via regression techniques has also been studied using several ML techniques. The debris flow type classification saw the comparison among compared RF, AdaBoost, and Gradient Boosting (GBDT) and found RF to be the best performing (Wang et al. 2019). Another area where ML has made significant progress is extraction and classification (Ghorbanzadeh et al. 2019), which is carried out either at pixel or object level. The success in the use of ML has made researchers emphasize on improving its performance for both the aspects (Moosavi et al. 2014; Mezaal et al. 2018).

Lately, there also has been a focus on the study of dynamics of landslides, like analysing the movement and volume estimation. As an example, Krkač et al. (2017) used RF technique to predict the movement of landslides and found that it has the capability to predict the evolution of daily displacements for up to 30 days. Li et al. (2018) highlighted in their study that the use of classical ML algorithms may fail to understand landslide movement in case of heavy precipitation and suggested the use of extreme learning machine (ELM) approach. 
However, these approaches fail to capture the long temporal dependency for the input sequence, thereby the use of long short-term memory (LSTM) neural network was proposed by Yang et al. (2019). Shirzadi et al. (2017) compared various statistical and machine learning models to determine the landslide volume and found ANFIS model to be outperforming the other models. The displacement of landslide as a measure of forecasting was studied by Liu et al. (2014) wherein three different ML models were tested (SVM, Relevance Vector Machine and Gaussian Process) and found GP to be the best performing. These studies are encouraging and highlights the use of ML models to understand the dynamic components of landslides which would help overcome the dependence towards physical models.

\subsubsection{Droughts}

The aim to predict the occurrence of any geohazard in both spatial and temporal context has fascinated researchers for a long time, and drought prediction is probably the biggest challenge on both the fronts. Unlike other geohazards, where the onset is known, droughts do not have a clear definition of its onset and is depended on who is defining it (LloydHughes, 2014). The use of ANN has proved to be quite successful in drought prediction due to the non-linear behaviour (Mishra and Singh, 2010; Dikshit et al. 2020c). The major breakthrough in drought forecasting has been the use of lagged climatic variables as predictors, and has seen an immense improvement in forecasting results, especially for longer lead times (Hao et al. 2018). At present, drought prediction has seen the use of several basic and advanced ML models. Mishra and Singh, (2011); AghaKouchak et al. (2015) and Hao et al. (2018) in their review articles on drought prediction provide details about the various drought modelling approaches used in the literature. These articles emphasize that future works should look towards developing more advanced models and to assimilate data at higher temporal scale. The assimilation of data at higher temporal scales could help to unearth new prospects like forecasting flash droughts (Pendergrass et al. 2020). This would also help immensely in understanding drought propagation, i.e., how rainfall shortage disseminate to a deficit in low streamflow and soil moisture along with the influence of anthropogenic activities affecting this transformation (Van Loon et al. 2016). We have seen how improvement in human behaviour pushed the Zero Day in Cape Town in 2018 to an indefinite period (Simpkins, 2018). We believe that the accurate temporal determination of drought propagation can be achieved within the next decade, due to the availability of higher temporal resolution data from both remote sensing perspective (Himawari 8) and climate perspective (ERA5) along with improved time series forecasting models, particularly in deep learning context. The development of more advanced tools would help to forecast drought at longer lead times, possibly multi-years, surpassing the present limitations of 12 months. 
The study by Sutanto et al. (2019) proposed a novel methodology of using RF model to predict the impact of drought. For regression tasks, focus has more on the use of knowledgebased processes (e.g., Analytic Hierarchy Process) and the use of ML is fewer compared to prediction tasks. The study by Rahmati et al. (2020) exploited this aspect and compared six different ML models to analyse drought risk of south-east Australia.

\subsubsection{Earthquakes}

For long, there have been many apprehensions about the possibility of predicting earthquakes. Nagerestani et al. (2002) used Back Propagation Neural Network (BPNN) and Liu et al. (2004) utilising Radial Basis Function (RBF) neural networks to predict earthquakes. However, the failure of predicting the 2004 Parkfield earthquake (Bakun et al. 2005) was a setback as it involved massive resources both in terms of money and manpower. The introduction of advanced ML algorithms in the field has created a new hope among researchers to find patterns which could help in predicting earthquakes days ago in the foreseeable future. For this, ANN and RF have been used extensively, e.g. - Rouet-Leduc et al., (2017) used RF to predict the remaining time before the next failure derived from earthquakes, whereas Buscema et al. (2015) used ANN to estimate the magnitude of the events recorded daily and proving it to be a promising technique. Martínez-Álvarez et al. (2011) used regression to forecast to analyse the temporal patterns for an earthquake prediction. We expect that the biggest breakthrough in geohazards field would be an accurate prediction of earthquakes, primarily because of the scale of damage both in terms of lives and economy.

ML has also been successful to assess damage post-earthquake event, like Weiland et al. (2016) used SVM to detect such changes post March 2011 earthquake event in Japan. Comparing SVM with ANN reflected similar results while classifying damages post September 2018 event of Indonesia (Syifa et al. 2019). The regression problems like analysing seismic vulnerability which majorly depends on magnitude prediction along with structural and geological aspects have seen ANN as an effective model because the inherent nature of earthquake phenomena can be uncertain (Vicente et al. 2011; Jena et al. 2020a). The integration of vulnerability with hazard and probability to develop risk maps is essential to quantify the population at risk, which is often carried out using multi-criteria decision making and analytic hierarchy process (AHP) (Jena et al. 2020b). This is certainly an area where ML could prove to be immensely helpful as AHP is heavily dependent on the expert opinions and is subjective.

\subsubsection{Floods and glacier lake outbursts}


The prediction of floods has majorly initially involved the use of single algorithms like ANN, SVM, neuro-fuzzy, Multilayer Perceptron (MLP) which has slowly moved to the use of hybrid models like adaptive neuro-fuzzy inference systems (ANFIS), Wavelet Neural Network (WNN). ANNs have been mostly used and its performance among statistical models and/or other ML based models have been very well tested. The studies reveal that although ANN exceeds the performance compared to traditional statistical models, however, hybrid models have overcome the difficulties of the use of single ANN based models and achieved better accuracy. For flood susceptibility, RF model has proven to be successful over other conventional ML models (Chen et al. 2019; 2020) and several works have encouraged its use, especially when assessing at a national scale (Zhao et al. 2018). Tehrany et al. (2015) analysed the efficiency of SVM technique using four different kernel types for flood susceptibility at north-eastern part of Malaysia. Rahman et al. (2019) integrated statistical, ML and AHP to identify flood hazard areas in Bangladesh as an alternative to the conventional approaches (hydraulic and hydrological) that involves several parameters. More recently, Wagenaar et al. (2020) provided a comprehensive review of ML models in response to flood risk and impact assessment. Their findings suggest the possible use of ML under different conditions of exposure, hazard and impact. The study also predicts the areas where the use of ML is likely or unlikely to increase.

The change anomaly-detection using ML has paved new ways in the field of glacier outbursts as it has been led to build an inventory for reliable Glacial Lake Outburst Flood (GLOF) hazard assessment. Veh et al. $(2018 ; 2019)$ used RF model to develop such an inventory for Himalayan region. Dirsherl et al. (2020) used RF to classify glacier types in Antarctic ice sheet which is first of its kind study conducted on a large scale.

\subsection{Hybrid Models}

However, the domains studied under each geohazard is different from each other, with some avenues being more explored compared to other. As an example, landslide susceptibility mapping has been relatively more studied compared to other aspects like hazard and risk. The use of traditional machine learning has proved to be quite useful in understanding several traits of geohazard studies. However, analysing the complex nature involved with the increase in sample size and diversity has hampered the use of shallow learning algorithms due to their finite cell limit (Wang et al. 2019). The way forward to overcome this concern is by the integration of ML algorithms known as hybrid models or the use of deep learning. In almost all the various specific domains, the use of hybrid models have preceded the use of a single algorithmic approach. Hybrid models are basically ensembles developed by integrating algorithms that do not belong to the same family (Kadavi et al. 2018). Sameen et al. (2020) used integrated regularised greedy forests (RGF) and RF models for regression 
and classification purposes for Bhutan Himalayas. Fanos and Pradhan, (2019) used hybrid model to predict probable rockfall source in Malaysia and achieved accuracy of 0.93 . Alizadeh et al. (2018) used a hybrid framework involving ANN with Analytic Network Process (ANP) to assess earthquake vulnerability in north-western part of Iran. Zhou et al. (2016) forecasted slope displacement using Swarm Optimisation and SVM coupling approach, and the result showed an improvement in predictive abilities. Asencio-Cortés et al. (2018) used several regression algorithms along with stacking-based ensemble learning approach to predict earthquake magnitude. Such kind of study gives hope to ensemble learning, especially in the future for big data analytics. The use of hybrid models in case of flood studies have overcome the difficulties faced by the use of single ANN based models and achieved better accuracy. Like, the ability to predict short-term flood up to $2 \mathrm{hr}$ can be accurately done using single algorithms, but hybrid algorithms are better to predict longterm floods (Mosavi et al. 2018). Mostly, ANFIS has been used for hybrid algorithms for flood regression studies (Tien Bui et al. 2018). In general, it can be said that the use of hybrid models has proven to be more useful in terms of accuracy compared to single algorithmic use, however its use and application is dependent on the input data respective to geohazard type and the hybrid model being considered.

\section{Deep Learning}

Deep learning is basically a representation learning method composed of several representation layers, obtained by constituting non-linear modules. Each of these modules transforms the representation at every level which enables to learn complex functions (LeCun et al. 2015). The benefit of using such models is its ability to learn from the data instead of handcrafting features based on domain expertise. The field of deep learning is pretty new to geohazard researchers and there are several architectures, each having its own benefits and shortcomings. We highlight only the majorly used architectures, for an extensive understanding, readers are referred to Shrestha and Mahmood, (2019) and Ball et al. (2017) wherein a comprehensive review is provided. The application of deep learning types in geohazards has been on segmentation (Baumhoer et al. 2019), feature extraction (Ci et al. 2019), detection (Sameen and Pradhan, 2019) and prediction (Hu et al. 2019). The various types of image segmentation with their benefits and limitations have been elaborately discussed in Minaee et al. (2020).

\subsection{Deep learning architectures}

\subsubsection{Auto-encoder (AE)}

Auto-encoder (AE) can be considered as a special kind of ANN which can learn useful features from unsupervised data by reducing the reconstruction error between input and 
output values of the network (Bengio et al. 2007). It comprises encoder and decoder phases that could transform an input to representation and also has the ability to transform back to original signal, with the number of layers in output and output being similar and larger than hidden units (Fig. 2a). Hinton and Salakhutdinov, (2006) emphasized that such a design could reduce nonlinear dimensionality. In situations of high dimensional input data, single layer $\mathrm{AE}$ is ineffective and multiple layers are stacked to build Stacked $\mathrm{AE}$ (SAE) architecture. There are several variants of $\mathrm{AE}$ each serving a specific purpose like stacked denoising which intakes a corrupted sample data and are trained to recover the perfect "repaired" input to robustly obtain a good representation (Vincent et al. 2010).

\subsubsection{Deep belief network (DBN)}

The primary component of DBN are the Restricted Boltzmann machines (RBM) which involves one visible input and one hidden output layer, a type of generative model (Hinton and Sejnowski, 1986). The stacking of multiple RBMs builds DBN, wherein the output of a hidden layer of the preceding RBM is used as input of a visible layer for the following RBM (Hinton et al. 2006). The training of DBN is similar to SAE wherein the initial parameters are learned in a greedy layer-wise unsupervised pattern and thereby fine tuning the parameters using an additional layer at the top. The connections between visible and hidden inputs are restricted, with no connections between the nodes at same layer implying the visible units to be independent, which leads to efficient unsupervised training schemes (Hinton, 2002). This can be considered being more efficient compared to a single hidden layer using greedy layer-wise manner, as relatively better hierarchical representations can be learned which could better reflect the latent structures in the input data (Fig. 2b).

\subsubsection{Convolutional neural network (CNN)}

$\mathrm{CNN}$ network can be considered being inspired by human visual cortex with the capability to automatically capture and learn the spatial feature hierarchies and comprising three building blocks: convolutional, pooling and fully-connected layers (LeCun et al. 1998) (Fig. 2c). The first block is responsible to extract the local features from various locations of the raw input or capture the intervening features with learnable filters known as kernels. These kernels can also be optimised depending on the given problem with the aim to reduce gap between actual and output layers through an optimization algorithm like backpropagation and gradient descent. The pooling layer can be considered as a subsampling layer which reduces the size of the input layer and specifies either the maximum or average value from each subarea of the preceding layer. Finally, after completing an alternate stacking of convolutional and pooling layers, one or more fully-connected layers are added for classification or feature representation purposes. When multiple CNNs are stacked for capturing local geometric or 
spatial patterns, it is known as deep CNN. Some popular CNN architectures are AlexNet (Krizhevsky et al. 2012), GoogLeNet (Szegedy et al. 2015), VGG (Simonyan and Zisserman, 2015), ResNet (He et al. 2016).

\subsubsection{Recurrent neural network (RNN)}

The sole purpose of RNN which is a type of neural networks is to analyse non-stationary processes like time series data (Williams and Zipser, 1989). It can be considered as a series of interconnected networks for time series analysis and can be trained using back propagation based gradient descent algorithms. RNN considers both the current input data and the preceding data to map target vectors, a sharp contrast to neural networks which map target vectors by multiplying weights (Fig. 2d). One of the key advantages of using RNN is its ability to store an internal memory of previous inputs in the network, which enables it to recall key events that occurred several times in the past and could prove to be important in situations which are depended on past events. The issue arises when multiple RNNs are stacked, which leads to vanishing and exploding gradient problems (Bengio et al. 1994). This concern was tackled by the introduction of long short-term memory (LSTM) (Hochreiter and Schmidhuber, 1997). It consists of a cell which would store the values to be used at random intervals and three gates, viz., input, output and forget gate, to control and adjust the cell state.

With the increased interest towards the application of deep learning, various open source frameworks have also been developed. Figure 3 shows the most popular open source deep learning platforms based on the number of stars received in GitHub. The detailed discussion about each framework and its comparisons can be found at De Felice, (2017). Readers are also referred to https://developers.google.com/machine-learning/glossary/ and http://www.wildml.com/deep-learning-glossary/ for a complete glossary.

\subsection{Application of DL on geohazard studies}

The application to specific problems in geohazard are still in the early days, but few works have shown their capability to outperform ML and depicting promising results. The works of Ji et al. (2019) to identify damage buildings post 2010 Haiti earthquake using CNN architecture and its further improvement by $\mathrm{Ci}$ et al. (2019) to classify damage into various degrees, and not just damaged or not damaged are good signals for earthquake researchers. On the other hand, Mangalathu and Burton, (2019) used LSTM method to classify building damage based on textual descriptions of damage. The textual descriptions was based on crowd-sourced information gathered from citizens and social media platforms. Such kind of studies could prove to be effective in mitigation purposes as post any geohazard event. A barrage of information is available, which often makes it difficult to assess the credibility and 
the associated threat level. The use of deep learning has proved to be crucial for classification purposes in glacier fronts (Baumhoer et al. 2019) which used a modified U-net segmentation to classify calving fronts along Antarctic coastline. Such study is crucial as our understanding on glacial retreat needs to be improved as glacier melting due to climate change, and the subsequent rise in sea-level rise is an imminent threat in the future.

Similarly, in the case of landslide detection and mapping, researchers have attempted various architectures to improve classification. The use of CNN to identify landslides was attempted by Chen et al. (2018) wherein they analysed the changes under multi temporal images and utilized spatio-temporal context analysis. Lei et al. (2019) developed a CNN model with pyramid pooling instead of global pooling for developing a landslide inventory. Their model could explore the context of images efficiently and better detect landslides on various scales. Ghorbanzadeh et al. (2019) compared various conventional ML models with $\mathrm{CNN}$ and highlighted the efficacy of deep learning provided hyper parameters are carefully optimized. Sameen and Pradhan, (2019) trained a residual networks_(ResNet) on spectral and topographic features, thereafter Prakash et al. (2020) used modified U-net segmentation along with ResNet to map landslide inventory.

The spatio-temporal prediction of floods was studied by $\mathrm{Hu}$ et al. (2019) wherein they developed a combination of novel LSTM model with Reduced Order Model (ROM) and the results were found to be complementary. The model was successfully able to analyse the spatio-temporal aspects, and such studies can help to predict floods assisting in near realtime predictions. For drought monitoring aspects, Shen et al. (2019) used a deep feedforward neural network for the prediction of Standardised Precipitation Evaporation Index (SPEI), a popular drought index at a regional scale in China and found a correlation with meteorological and agricultural droughts. The study by Wang et al. (2019) shows promising results on the use of $\mathrm{CNN}$ architecture for regression purposes for landslide susceptibility, which outperformed optimized SVM model. Pedro et al. (2018) attempted to detect earthquake by training labelled raw data with supervised CNNs to extract features that can efficiently differentiate seismic signals from seismic noise.

The use of DL in geohazard studies is still in infancy, with some prominent works in feature extraction and classification. The recent work by Pradhan et al. (2020) on the use of CNN for feature extraction and Word2Vec model to assign class attributes eould-proved to be immensely helpful for change detection studies., especially in case of earthquakes and glaciers.Figure 4 highlights the various possible domains of geohazard studies and showing the trend in the possible studies towards achieving a data-driven environment. A more thorough understanding of other aspects like spatio-temporal modelling and improving predictive capability is bound to happen in the near future. 
One of the main differences between computer vision researchers and geohazard researchers is the vast difference in dataset availability and variants in classification. One way to understand it, is the ImageNet classification, which contains a database of images labelled by humans, like 'cat' or 'dog'. In such cases, there is a definitive answer to the labelling case, which may not true for geohazard community. For e.g. - labelling earthquake damages depending on the level of damage depends on a defined set of rules, which may or may not hold true for all parts of the world. Similarly, labelling a drought event can be tricky as it depends on the objectives of the study and the trends used to identify the drought. Also, usually it is difficult to find a set pattern among any recurring geohazards, like two drought events or earthquake events are not similar and most of the times the results determined need an external validation, from the premise which would provide the actual accuracy of the model. Also, images used in computer vision field typically have 3 bands (RGB), but hyperspectral images extend to hundreds of spectral channels well beyond the visible range, which often induce different statistical properties to those of natural images.

\section{What have we learnt?}

It can be well accepted that the use of highly advanced computational algorithms can solve several challenging aspects for every geohazard application. However, its use in earthquake and GLOF has been very minimal, therefore it's suggested that more algorithms needs to be tested in understanding aspects like movement and volume associated with GLOF or predictive capability of earthquakes. Also, the credibility of the results irrespective of the study conducted is highly dependent on the quality of the dataset and the predisposing factors being considered, which has been aptly pointed out in several studies (Dikshit et al. $2020 b)$. Therefore, in terms of data requirements, efforts should be made towards the use of higher spatial resolution and all the relevant causative factors. However, there may be still many parts of the world where higher resolution dataset may not be available, which has led several researchers to use the freely available Google Earth images.

The modelling aspects tell us that use of ML algorithms are well established, and the trend is more towards the use of hybrid models or comparing different single based ML algorithms along with ensemble techniques to determine the best modelling approach. This has been vastly used in terms of spatio-temporal modelling and regression techniques. Among the single based algorithmic approach, the most used models are RF, ANN, LR, SVM, decision trees and neuro-fuzzy. The use of better optimisation techniques has also been on a rise which could help in improving our understanding of the subject. One interesting aspect is the use of statistical tools (equal breaks, quantile, natural breaks) for reclassifying maps developed using various regression techniques, especially in the field of landslides and floods, thereby suggesting to use ML techniques for reclassifying maps. 
One of the major problems with ML is its ability to identify the subtleties due to the issue of insufficient data. This is where deep learning approaches can play an important role and is evident that it will surpass the accuracy obtained from ML based models in every aspect. The collection of enormous data to understand the correspondence between factors and the respective geohazard has been one of the concerns which researchers have tried to address. The use of either machine learning or deep learning approaches to study various areas of geohazards can be immensely complemented with the use of physical-based models. The use of physical-based models has their own challenges primarily owing to the in-depth knowledge and expertise of various parameters involved. The use of ML models in combination with physical models is one area which deserves more attention. The integration is required as the sole use of physical models has failed in several aspects. Generally, it has been used in tandem with statistical models (Goetz et al. 2011) and the focus should be on using ML models. Bellos and Tsakiris, (2016) coupled physical based 2D hydrodynamic model with hydrological unit graph theory which improved flood modelling for catchment area in Greece. the flood physieal models. This was further highlighted by Felder et al. (2017) which combined hydrodynamic and hydrological models which helped in improving the physical aspects of probable maximum flood.

\section{The future ahead}

There are several fronts which needs improvement for better understanding in every aspect of geohazards ranging from data availability, computational challenges, integration with physical models, application on a larger scale, interpretability of label features along with the ability to develop robust models to analyse multi hazard challenges. Some of the most pressing factors to be included in the future geohazard studies would be the effect of climate change and anthropogenic activities. The concerns about climate change and their influence on geohazard has been aptly highlighted in reviews discussing its effect on landslides (Gariano and Guzzetti, 2016), droughts (Ault, 2020), GLOF (Harrison et al. 2017). The causative factors in all the studied geohazards, except earthquakes, should include the factor of human activities, as it's increasingly becoming an important aspect (Gill and Malamud, 2017).

Here, we emphasize the need towards understanding consecutive geohazard events which are on a rise and will continue in the foreseeable future due to the ever changing climatic and anthropogenic landscape. Few recent examples of such events are the April 2015 Nepal earthquake, 2018 summer of Japan or the 2019 Australia summer. Zuccaro et al. (2018) describes a theoretical model for cascading effect scenario analysis and the basic framework towards identifying elements at risk. The Sendai Framework for Disaster Risk Reduction (UNDRR, 2015) and the Paris Agreement (UNFCCC, 2015) also calls for development 
towards formulating a comprehensive risk assessment framework. De Ruiter et al. (2020) attempted to fill this gap and provided the fundamental knowledge about multi-risk assessments. The general focus has been towards multi-hazard approaches which is a part of consecutive events and there are four key factors that needs to be considered while addressing consecutive events (Gill and Malamud, 2014): a) comparing various individual geohazards for a definite region b) considering all the various possible interactions between the geohazards c) impact of spatio-temporal interaction among hazards d) dynamic vulnerability and their effect on societal aspects.

Most of the studies have considered the first two factors and neglected the impacts of hazards which may coincide with spatially and/or temporally along with dynamic vulnerability aspects. The works on multi-hazard probability assessment is a small step towards our understanding of consecutive events and can be improved with the use of hybrid models or deep learning architecture. We recommend that researchers should emphasize towards improving the understanding of the possibility of such events and not solely focus on multi-hazard scenarios. The general scenario is analysing the coupling process, and the mechanism involved for a single geohazard event occurrence. There is an emergence towards collecting a vast pool of data at a higher temporal scale either based on sensor-based monitoring system or satellite imagery like Himawari 8 . The future would gradually move towards geostationary satellites images (Sawada et al. 2019) and/or climatic datasets like the ERA5 reanalysis data (C3S, 2017). The emergence of xBD dataset (Gupta et al. 2019) which includes 700,000 building damage labels corresponding to eight different disaster types is an encouraging step which can help in developing more novel methodologies. The focus for future studies should be on determining the possible scenarios after a hazard has occurred and this can be achieved by the amalgamation of data driven models and physical modelling approaches (Figure 5).

The trade-off between the prediction and explanation with DL models is a challenge with recent advances in interpretable models like post-hoc interpretability techniques (Rai, 2020). However, a consensus needs to be built on what domains are necessary for interpretability in geohazards. It's almost certain that prediction of an event needs a high level of explainability, but the accuracy in change anomaly topics while preparing inventory or regression techniques like sustainability is an open-ended question. We believe that studies such as damage assessment post a geohazard event, determining the temporal dynamics like landslide flow length or mapping flooded areas and segmentation problems need high accuracy and explainability should not be an issue.

\section{Conclusions and Future Recommendations}


The availability of large sums of data and our improved computational capability has made researchers focus towards the use of machine learning and subsequently deep learning. It is evident that some geohazard type (landslides, floods, drought) have benefitted more compared to other types (glacier outbursts). The challenges towards a better understanding of geohazards are enormous and therefore, we recommend the following:

1) The studies have focussed towards single hazards and therefore the focus should shift towards understanding consecutive event case scenarios using data-driven approaches. A step-by-step process should be followed towards this, wherein studies like comparison with ground-based data and remote sensing data should be analysed. There will be several key areas across the globe where either of the data collection methods would not give reliable results, which would make the optimisation techniques more significant.

2) The emergence of deep learning towards understanding the complex spatio-temporal relationships among variables is an encouraging step. However, its use across multi scales and sources are still limited which prevents it from using it frequently especially on a large scale (regional or national). These challenges are expected to be overcome in the near future with the availability of climatic dataset (ERA5), multi-hazard database (xBD) and higher temporal remote sensing data (Himawari 8). The future of geohazards could become a data problem similar to geosciences and therefore robust models need to be prepared which could aptly serve the purpose of understanding the mechanism involved behind a geohazard event.

3) Machine learning approaches use certain approximations which are considered being valid or are good approximations irrespective of the study being performed. Deep learning has the ability to overcome these issues and reckon that in future it will be the leading method for several geohazard related studies. It would also give an opportunity to researchers to tackle the issue of limited data either spatially or temporally, which can be very crucial for regions suffering from data availability.

4) Currently, even though ML or DL approaches help in spatio-temporal modelling, the physical relevance is not well-known. Few works have attempted to couple statistical models or used sensor-based instruments to understand the involved mechanism. However, the parameters used should work towards providing a sense of physical interpretation. The issue of uncertainty and extrapolation should be considered generating more confidence in the models used. The emergence of new datasets can help couple data driven models with physical models can help in a better understanding of the process. Such a process could eventually help in achieving explainable and interpretable ML models (Gunning, 2017), along with a combination of logic rules with deep neural networks (Reichstein et al. 2019). 
5) The evident change in climate and increase in anthropogenic activities would make consecutive events a usual norm in the future. Therefore, it becomes very significant working towards forecasting the possible geohazards after an event has occurred at different time scales (like possible events after few days, weeks or months) and can be very well complemented with physical models. This would also help in identifying the elements at risk, which could help in better mitigation strategies.

\section{Acknowledgements}

The study was financially supported by the Centre for Advanced Modelling and Geospatial Information Systems (CAMGIS), Faculty of Engineering and Information Technology, University of Technology Sydney (UTS). This research was also supported by Researchers Supporting Project number RSP-2020/14, King Saud University, Riyadh, Saudi Arabia. Authors would also like to thank the Professor M. Santosh for his editorial comments and anonymous reviewers for their careful review of the original manuscript and their valuable suggestions which helped us to improve the manuscript.

\section{References:}

AghaKouchak, A., Farahmand, A., Melton, F., Teixeira, J., Anderson, M., Wardlow, B., Hain, C. (2015). Remote sensing of drought: Progress, challenges and opportunities. Reviews of Geophysics, 53, 452-480.

Alizadeh, M., Ngah, I., Hashim, M., Pradhan, B., Pour, A.B. (2018) A Hybrid Analytic Network Process and Artificial Neural Network (ANP-ANN) Model for Urban Earthquake Vulnerability Assessment. Remote Sens., 10, 975.

Asencio-Cortés G., Morales-Esteban A., Shang X., et al. (2018) Earthquake prediction in California using regression algorithms and cloud-based big data infrastructure. Comput. Geosci., 115, 198-210

Ault, T.B. (2020) On the essentials of drought in a changing climate. Science, 368 (6488), 256-260

Bakun, W., Aagaard, B., Dost, B. et al. (2005) Implications for prediction and hazard assessment from the 2004 Parkfield earthquake. Nature 437, 969-974

Ball, J. E., Anderson, D. T., Chan, C. S. (2017) Comprehensive survey of deep learning in remote sensing: theories, tools, and challenges for the community. J. Appl. Remote Sens. 11, 042609 
Barua, S., Ng, A., Perera, B. (2012) Artificial neural network-based drought forecasting Antarctic Glacier and Ice Shelf Fronts from Sentinel-1 Imagery Using Deep Learning. Remote Sens., 11, 2529.

Bellos, V., Tsakiris, G. (2016) A hybrid method for flood simulation in small catchments combining hydrodynamic and hydrological techniques. J. Hydrol., 540, 331-339.

Bengio, Y., Simard, P., Frasconi, P. (1994) Learning long-term dependencies with gradient descent is difficult. IEEE Trans. Neural Netw., 5, 157-166

Bengio, Y., Lamblin, P., Popovici, C., Larochelle, H. (2007) Greedy layer-wise training of deep networks. Advances in Neural Information Processing Systems, 153-160

Bishop, C. M. (2006) Pattern recognition and machine learning. Berlin: Springer.

Buczak, A.L., Guven, E. (2016) A Survey of Data Mining and Machine Learning Methods for Cyber Security Intrusion Detection. IEEE Commun. Surv. Tutor., 18 (2), 1153-1176

Chen, W., Xie, X., Wang, J., Pradhan, B. et al. (2017) A comparative study of logistic model tree, random forest, and classification and regression tree models for spatial prediction of landslide susceptibility. Catena, 151, 147-160

Chen, K.T., Prishchepov, A.V., Fensholt, R., Sabel, C.E. (2019) Detecting and monitoring long-term landslides in urbanized areas with nighttime light data and multi-seasonal Landsat imagery across Taiwan from 1998 to 2017. Remote Sens. Environ., 255, 317-327

Chen, W., Li, Y., Xue, W. et al. (2020) Modeling flood susceptibility using data-driven approaches of naïve Bayes tree, alternating decision tree, and random forest methods. Sci. Total Environ., 701, 134979

Ci, T., Liu, Z., Wang, Y. (2019) Assessment of the Degree of Building Damage Caused by Disaster Using Convolutional Neural Networks in Combination with Ordinal Regression. Remote Sens., 11, 2858

Collobert, R., et al. (2011) Natural language processing (almost) from scratch. J. Mach. Learn. Res. 12, 2493-2537.

Copernicus Climate Change Service (C3S) (2017) ERA5: Fifth generation of ECMWF atmospheric reanalyses of the global climate. Copernicus Climate Change Service Climate Data Store (CDS), https://cds.climate.copernicus.eu/cdsapp\#!/home 
Cooner, J.A., Shao, Y., Campbell, B.J. (2016) Detection of urban damage using remote Sens., 8, 868

Dang, V., Dieu, T.B., Tran, X. et al. (2019) Enhancing the accuracy of rainfall-induced landslide prediction along mountain roads with a GIS-based random forest classifier. Bull Eng Geol Environ 78, 2835-2849

De Felice, M. (2017) Which deep learning network is best for you? IDG Communications, [online] Available: http://www.cio.com/article/3193689/artificial-intelligence/which-deeplearning-network-is-best-for-you.html.

De Ruiter, M.C., Couasnon, A., van den Homberg, M.J.C. et al. (2020) Why We Can No Longer Ignore Consecutive Disasters. Earth's Future, 8, 3, e2019EFo01425

Dikshit, A., Sarkar, R., Pradhan, B., et al. (2020a) Temporal Probability Assessment and Its Use in Landslide Susceptibility Mapping for Eastern Bhutan. Water 2020, 12, 267.

Dikshit, A., Sarkar, R., Pradhan, B., et al. (2020b) Rainfall Induced Landslide Studies in Indian Himalayan Region: A Critical Review. Appl. Sci., 10, 2466.

Dikshit, A., Pradhan, B., Alamri, A.M. (2020c) Short-Term Spatio-Temporal Drought Forecasting Using Random Forests Model at New South Wales, Australia. Appl. Sci., 10, 4254 .

Dirscherl, M., Dietz, A.J., Kneisel, C., Kuenzer, C. (2020) Automated Mapping of Antarctic Supraglacial Lakes Using a Machine Learning Approach. Remote Sens., 12, 1203.

Fanos, A.M., Pradhan, B. (2019) A Novel Hybrid Machine Learning-Based Model for Rockfall Source Identification in Presence of Other Landslide Types Using LiDAR and GIS. Earth Syst Environ 3, 491-506

Farabet, C., Couprie, C., Najman, L. \& LeCun, Y. (2012) Scene parsing with multiscale feature learning, purity trees, and optimal covers. In Proc. International Conference on Machine Learning http://arxiv.org/abs/1202.2160

Feldar, G., Zischg, A., Weingartner, R. (2017) The effect of coupling hydrologic and hydrodynamic models on probable maximum flood estimation. J. Hydrol., 550, 157-165

Felicísimo, Á.M., Cuartero, A., Remondo, J. et al. (2013) Mapping landslide susceptibility with logistic regression, multiple adaptive regression splines, classification and regression trees, and maximum entropy methods: a comparative study. Landslides 10, 175-189 
Gariano, S.L., Guzzetti, F. (2016) Landslides in a changing climate. Earth-Science Rev., 162, 227-252

Ghorbanzadeh, O., Blaschke, T., Gholamnia, K., Meena, S.R., Tiede, D., Aryal, J. (2019) Evaluation of Different Machine Learning Methods and Deep-Learning Convolutional Neural Networks for Landslide Detection. Remote Sens., 11, 196.

Gill, J.C., Malamud, B.D. (2014) Reviewing and visualizing the interactions of natural hazards. Reviews of Geophysics, 52, 680-722

Gill, J.C., Malamud, B.D. (2017) Anthropogenic processes, natural hazards, and interactions in a multi-hazard framework. Earth-Science Reviews, 166, 246-269

Goetz, J.N., Guthrie, R.H., Brenning, A. (2011) Integrating physical and empirical landslide susceptibility models using generalized additive models. Geomorphology 129:376-386.

Grace, K., Salvatier, J., Dafoe, A., Zhang, B. \& Evans, O. (2017) When will AI exceed human performance? Evidence from AI experts. arXiv Preprint arXiv:1705.08807

Gunning, D. (2017) Explainable Artificial Intelligence (XAI)

Gupta, R., Goodman, B., Patel, N., et al. (2019) Creating xbd: A dataset for assessing building damage from satellite imagery. In Proceedings of the CVPR Workshops, Long Beach, CA, USA, 16-20 June 2019

Hao, Z., Singh, V.P., Xia, Y. (2018) Seasonal Drought Prediction: Advances, Challenges, and Future Prospects. Reviews of Geophysics, 56(1):108-141

Harrison, S., Kargel, J.S., Huggel, C. et al. (2017) Climate change and the global pattern of moraine-dammed glacial lake outburst floods. The Cryosphere, 12, 1195-1209

He, K., Zhang, X., Ren, S., Sun, J. (2016) Deep residual learning for image recognition. Proceedings of the IEEE Conference on Computer Vision and Pattern Recognition, 770-778

Hinton, G.E., Sejnowski, T.J. (1986) Learning and Relearning in Boltzmann Machines. Parallel Distributed Processing: Explorations in the Microstructure of Cognition 1, 282-317

Hinton, G.E. (2002) Training products of experts by minimizing contrastive divergence. Neural Comput., 14, 1771-1800

Hinton, G.E., Salakhutdinov, R.R. (2006) Reducing the dimensionality of data with neural networks. Science, 313, 504-507

Hinton, G.E., Osindero, S., Teh, Y.W. (2006) A fast learning algorithm for deep belief nets. Neural Comput., 18, 1527-1554 
Hochreiter, S., Schmidhuber, J. (1997) Long short-term memory. Neural Comput., 9, 17351780

Hosny, A., Parmar, C., Quackenbush, J. et al. (2018) Artificial intelligence in radiology. Nat Rev Cancer 18, 500-510

Hu, R., Fang, F., Pain, C.C., Navon, I.M. (2019) Rapid spatio-temporal flood prediction and uncertainty quantification using a deep learning method. J. Hydrol. 575, 911-920

Huang, Y., Zhao, L. (2018) Review on landslide susceptibility mapping using support vector machines. Catena, 165, 520-529

Jena, R., Pradhan, B., Beydoun, G. (2020a) Earthquake vulnerability assessment in Northern Sumatra province by using a multi-criteria decision-making model. Int. J. Disaster Risk Reduct., 46, 101518

Jena, R., Pradhan, B., Beydoun, G. et al. (2020b) Integrated model for earthquake risk assessment using neural network and analytic hierarchy process: Aceh province, Indonesia. Geoscience Frontiers, 11(2), 613-634

Ji, M., Liu, L., Du, R., Buchroithner, M.F. (2019) A Comparative Study of Texture and Convolutional Neural Network Features for Detecting Collapsed Buildings After Earthquakes Using Pre- and Post-Event Satellite Imagery. Remote Sens., 11, 1202

Jordan, M.I., Mitchell, T.M. (2015) Machine learning: trends, perspectives, and prospects. Science, 349, 255-260

Kadavi, P., Lee, C.W., Lee, S. (2018) Application of ensemble-based machine learning models to landslide susceptibility mapping. Remote Sensing, 10, 1252

Krizhevsky, A., Sutskever, I. \& Hinton, G. (2012) ImageNet classification with deep convolutional neural networks. In Proc. Advances in Neural Information Processing Systems 25 1090-1098.

Krkač, M., Špoljarić, D., Bernat, S. et al. (2017) Method for prediction of landslide movements based on random forests. Landslides 14, 947-960

Lary, D.J., Alavi, A.H., Gandomi, A.H., Walker, A.L. (2016) Machine learning in geosciences and remote sensing. Geoscience Frontiers, 7(1):3-10

LeCun, Y., Bengio, Y., Hinton, G. (2015) Deep learning. Nature 521, 436-444

LeCun, Y., Bottou, L., Bengio, Y., Haffner, P. (1998) Gradient-based learning applied to document recognition. Proc. IEEE, 86, 2278-2324 
Lei, T., Zhang, Y., Lv, Z. et al. (2019) Landslide inventory mapping from bitemporal images using deep convolutional neural networks. IEEE Geosci. Remote Sens. Lett., 16, 6, 982-986

Liu, Z., Shao, J., Xu, W. et al. (2014) Comparison on landslide nonlinear displacement analysis and prediction with computational intelligence approaches. Landslides 11, 889-896 Lloyd- Hughes B. (2014) The impracticality of a universal drought definition. Theor Appl Climatol, 117:607-611

Mangalathu, S., Hwang, S.H., Choi, E. et al. (2019) Rapid seismic damage evaluation of bridge portfolios using machine learning techniques. Engineering Structures, 201, 109785

Mangalathu, S., Burton, H.V. (2019) Deep learning-based classification of earthquakeimpacted buildings using textual damage descriptions. Int. J Disaster Risk Reduction, 36, 101111

Mangalathu, S., Sun, H., Nweke, C.C., Yi, Z., Burton, H.V. (2020) Classifying earthquake damage to buildings using machine learning. Earthq. Spectra, 36(1), 183-208

Martínez-Álvarez F., Troncoso A., Morales-Esteban A., Riquelme J.C. (2011) Computational Intelligence Techniques for Predicting Earthquakes. In: Corchado E., Kurzyński M., Woźniak M. (eds) Hybrid Artificial Intelligent Systems. HAIS 2011. Lecture Notes in Computer Science, vol 6679. Springer, Berlin, Heidelberg

Mezaal, M., Pradhan, B., Rizeei, H. (2018) Improving landslide detection from airborne laser scanning data using optimized Dempster-Shafer. Remote Sens., 10, 1029.

Minaee, S., Boykov, Y., Porikli, F., et al. (2020) Image Segmentation Using Deep Learning: A Survey. https://arxiv.org/abs/2001.05566

Mishra A., Desai, V., Singh, V. (2007) Drought forecasting using a hybrid stochastic and neural network model. J. Hydrol. Eng., 12,626-638

Mishra, A. K., Singh, V. P. (2011) Drought modelling - A review. J. Hydrol., 403(1-2), 157175

Mnih, V., Kavukcuoglu, K., Silver, D. et al. (2015) Human-level control through deep reinforcement learning. Nature 518, 529-53

Moosavi, V., Talebi, A., Shirmohammadi, B. (2014) Producing a landslide inventory map using pixel-based and object-oriented approaches optimized by Taguchi method. Geomorphology, 204, 646-656 
Mosavi, A., Ozturk, P., Chau, K.-W. (2018) Flood Prediction Using Machine Learning Models: Literature Review. Water, 10, 1536.

Moravc``́k, M. et al. (2017) DeepStack: expert-level artificial intelligence in heads-up nolimit poker. Science 356, 508-513

Nefeslioglu, H.A., Gokceoglu, C., Sonmez, H. (2008) An assessment on the use of logistic regression and artificial neural networks with different sampling strategies for the preparation of landslide susceptibility maps. Engineering Geology, 97, 171-191

Pendergrass, A.G., Meehl, G.A., Pulwarty, R. et al. (2020) Flash droughts present a new challenge for subseasonal-to-seasonal prediction. Nat. Clim. Chang. 10, 191-19

Perol, T., Gharbi, M., Denolle, M. (2018) Convolutional neural network for earthquake detection and location. Sci. Adv., 4, e1700578

Pradhan, B., Lee, S. (2010) Landslide susceptibility assessment and factor effect analysis: backpropagation artificial neural networks and their comparison with frequency ratio and bivariate logistic regression modelling. Environment Modelling \& Software, 25(6):747-759

Pradhan, B., Al-Najjar, H.A.H., Sameen, M.I. et al. (2020) Unseen Land Cover Classification from High-Resolution Orthophotos Using Integration of Zero-Shot Learning and Convolutional Neural Networks. Remote Sens., 12, 1676.

Prakash, N., Manconi, A., Leow, S. (2020) Mapping Landslides on EO Data: Performance of Deep Learning Models vs. Traditional Machine Learning Models. Remote Sensing, 12, 346

Rahman, M., Ningsheng, C., Islam, M.M. et al. (2019) Flood Susceptibility Assessment in Bangladesh Using Machine Learning and Multi-criteria Decision Analysis. Earth Syst Environ 3, 585-601

Rahmati O., Falah, F., Dayal, K.S. et al. (2020) Machine learning approaches for spatial modeling of agricultural droughts in the south-east region of Queensland Australia. Sci. Total Environ., 699, 134230

Rai, A. (2020) Explainable AI: from black box to glass box. J. of the Acad. Mark. Sci. 48, $137-141$

Reichenbach, P., Rossi, M., Malamud, B.D., Mihir, M., Guzzetti, F. (2018) A review of statistically-based landslide susceptibility models. Earth-Sci. Rev., 180, 60-91

Reichstein, M., Camps-Valls, G., Stevens, B. et al. (2019) Deep learning and process understanding for data-driven Earth system science. Nature 566, 195-204 
Sameen, M.I. Pradhan, B. (2019) Landslide Detection Using Residual Networks and the unsupervised factor optimisation and regularised greedy forests. Computers \& Geosciences, 134,104336

Sawada, Y., Okamoto, K., Kunii, M., Miyoshi, T. (2019) Assimilating every-10-minute Himawari-8 infrared radiances to improve convective predictability. J. Geophys. Res. Atmos., 124, 2546-2561

Shirzadi, A., Shahabi, H., Chapi, K., et al. (2017) A comparative study between popular statistical and machine learning methods for simulating volume of landslides. Catena 157:213-226

Shen, R., Huang, A., Li, B., Guo, J. (2019) Construction of a drought monitoring model using deep learning based on multi-source remote sensing data. Int. J. Appl. Earth Obs. Geoinformation 79, 48-57

Shrestha, A. Mahmood, A., (2019) Review of deep learning algorithms and architectures, IEEE Access, 7, 53040-53065

Silver, D. et al. (2016) Mastering the game of Go with deep neural networks and tree search. Nature 529, 484-489

Simonyan, K., Zisserman, A. (2015) Very deep convolutional networks for large-scale image recognition. Proceedings of the ICLR

Simpkins, G. (2018) Running dry. Nature Clim Change 8, 369

Sutanto, S.J., van der Weert, M., Wanders, N. et al. (2019) Moving from drought hazard to impact forecasts. Nat Commun 10, 4945.

Sutton, R.S., Barto, A.G. (2018) Reinforcement learning: An introduction. MIT Press, Cambridge, MA

Syifa, M., Kadavi, P.R., Lee, C.-W. (2019) An Artificial Intelligence Application for PostEarthquake Damage Mapping in Palu, Central Sulawesi, Indonesia. Sensors, 19, 542

Szegedy, C., Liu, W., Jia, Y. et al. (2015) Going deeper with convolutions Proceedings of the IEEE Conference on Computer Vision and Pattern Recognition, 1-9

Tien Bui, D., Tuan, T.A., Klempe, H., Pradhan, B., Revhang, I (2016) Spatial prediction models for shallow landslide hazards: a comparative assessment of the efficacy of support 
vector machines, artificial neural networks, kernel logistic regression, and logistic model tree. Landslides 13, 361-378

Tien Bui, D., Khosravi, K., Li, S., et al. (2018) New Hybrids of ANFIS with Several Optimization Algorithms for Flood Susceptibility Modeling. Water, 10, 1210.

Tehrany, M.S., Pradhan, B., Mansoor, S., Ahmad, N. (2015) Flood susceptibility assessment using GIS-based support vector machine model with different kernel types. Catena, 125, 91101

UNDRR (2015). Sendai framework for disaster risk reduction 2015-2030. Retrieved from https://www.unisdr.org/files/43291 sendaiframeworkfordrren.pdf

UNFCCC (2015) Adoption of the Paris Agreement. Report No. FCCC/CP/2015/L.9/Rev.1, http://unfccc.int/resource/docs/2015/cop21/eng/logro1.pdf

Van Loon, A.F. et al. (2016) Drought in the Anthropocene. Nat. Geosci., 9, 89-91

Veh, G., Korup, O., Roessner, S., Walz, A. (2018) Detecting Himalayan glacial lake outburst floods from Landsat time series. Remote Sens. Environ., 207, 84-97

Veh, G., Korup, O., von Specht, S. et al. (2019) Unchanged frequency of moraine-dammed glacial lake outburst floods in the Himalaya. Nat. Clim. Chang. 9, 379-383

Vicente, R., Parodi, S., Lagomarsino, S., Varum, H., Silva, J.M. (2011) Seismic vulnerability and risk assessment: Case study of the historic city centre of Coimbra, Portugal. Bull. Earthq. Eng., 9, 1067-1096

Vincent, P., Larochelle, H., Lajoie, I. et al. (2010) Stacked denoising autoencoders: learning useful representations in a deep network with a local denoising criterion. J. Mach. Learn. Res., 11, 3371-3408

Wagenaar, D., Curran, A., Balbi, M., et al. (2020) Invited perspectives: How machine learning will change flood risk and impact assessment, Nat. Hazards Earth Syst. Sci., 20, $1149-1161$

Wang, N., Cheng, W., Zhao, M. et al. (2019) Identification of the Debris Flow Process Types within Catchments of Beijing Mountainous Area. Water, 11, 638.

Wang, Y., Fang, Z., Hong, H. (2019) Comparison of convolutional neural networks for landslide susceptibility mapping in Yanshan County, China. Sci. Total Environ., 666, 975993

Wang, H., Zhang, L., Yin, K. et al. (2020) Landslide identification using machine learning. https://doi.org/10.1016/i.gsf.2020.02.012 
Wieland, M., Liu, W., Yamazaki, F. (2016) Learning Change from Synthetic Aperture Radar Tsunami-Induced Changes. Remote Sens., 8, 792

Williams, R.J., Zipser, D. (1989) A learning algorithm for continually running fully recurrent neural networks. Neural Comput., 1, 270-280

Xiong, K., Adhikari, B.R., Stamatopoulos, C.A., et al. (2020) Comparison of Different Machine Learning Methods for Debris Flow Susceptibility Mapping: A Case Study in the Sichuan Province, China. Remote Sens., 12, 29

Xu, C., Dai, F., Xu, X., Lee, Y.H. (2012) GIS-based support vector machine modeling of earthquake-triggered landslide susceptibility in the Jianjiang River watershed, China. Geomorphology, 145-146, 70-80

Yang, B., Yin, K., Lacasse, S. et al. (2019) Time series analysis and long short-term memory neural network to predict landslide displacement. Landslides 16, 677-694

Yariyan, P., Avand, M., Soltani, F., et al. (2020) Earthquake Vulnerability Mapping Using Different Hybrid Models. Symmetry, 12, 405.

Zhao, G., Pang, B., Xu, Z. et al. (2018) Mapping flood susceptibility in mountainous areas on a national scale in China. Sci. Total Environ., 615, 1133-1142

Zhou, C., Yin, K., Cao, Y., Ahmed, B. (2016) Application of time series analysis and PSOSVM model in predicting the Bazimen landslide in the Three Gorges Reservoir, China. Engineering Geology, 204, 108-120

Zuccaro, G., De Gregorio, D., Leone, M. (2018) Theoretical model for cascading effects analyses. Int. Journal of Disaster Risk Reduction, 30, 199-215 


\section{Figure captions}

Figure 1: Various aspects of AI.

Figure 2: Various Deep Learning architectures: (a) Auto-encoder, (b) Deep belief network, (c) Convolutional neural networks, and (d) Recurrent neural networks.

Figure 3: Popular deep learning frameworks based on GitHub count as of April, 2020.

Figure 4: Flowchart highlighting the various aspects of geohazard studies and the current focus towards models used along with the future scenario.

Figure 5: Flowchart highlighting the current practices and the future aspects expected in order to achieve better intelligence quotient and gradual understanding towards understanding of the mechanism and interpretability providing "Explainable AI".

\section{Table captions}

Table 1: Strengths and limitations of various deep learning methods in various geohazard studies. 


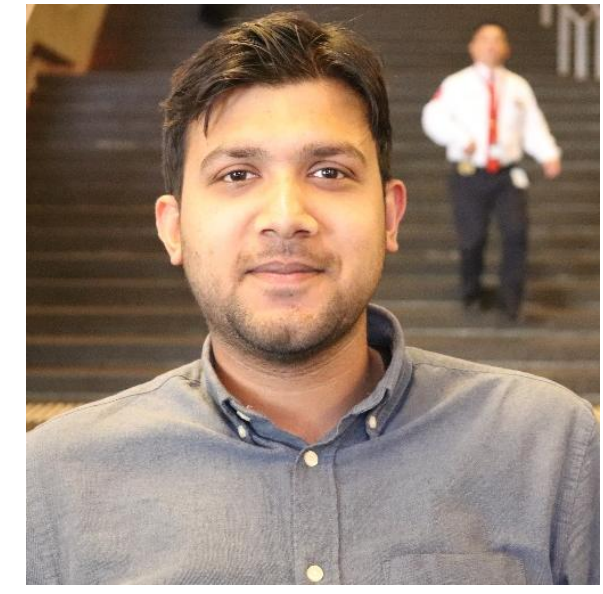

ABHIRUP DIKSHIT is a PhD student at the Centre for Advanced Modelling and Geospatial Information Systems (CAMGIS), University of Technology, Sydney. He has completed his Master (MS by Research) in Civil Engineering from the International Institute of Information Technology (IIIT) Hyderabad, India. He was awarded the International Research Training Program Scholarship (IRTP) by the Australian Government to pursue his doctoral research.

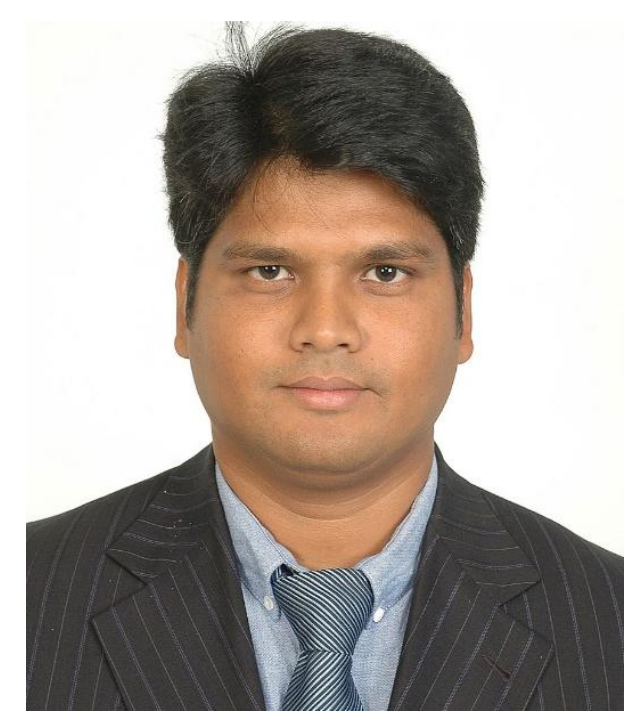

BISWAJEET PRADHAN received the Habilitation in remote sensing from the Dresden University of Technology, Germany, in 2011. He is founding Director of the Centre for Advanced Modelling and Geospatial Information Systems (CAMGIS), Faculty of Engineering and IT. He is also the Distinguished Professor with the University of Technology Sydney. He is also an internationally established Scientist in the fields of geospatial information systems (GIS), remote sensing and image processing, complex modeling/geo-computing, machine learning and soft-computing applications, natural hazards, and environmental modeling. Since 2015, he has been serving as the Ambassador Scientist for the Alexander Humboldt Foundation, Germany. Out of his more than 450 articles, more than 400 have been published in science citation index (SCI/SCIE) technical journals. He has authored eight books and 13 book chapters. He was a recipient of the Alexander von Humboldt Fellowship from Germany. He received 55 awards in recognition of his excellence in teaching, service, and research, since 2006. He was also a recipient of the Alexander von Humboldt Research 
Fellowship from Germany. From 2016 to 2019, he was listed as the World's Most Highly Cited Researcher by Clarivate Analytics Report as one of the world's most influential mind. In 2018 and 2019, he was awarded as the World Class Professor by the Ministry of Research, Technology and Higher Education, Indonesia. He is also an Associate Editor and an Editorial Member of more than eight ISI journals. He has widely travelled abroad, visiting more than 55 countries to present his research findings.

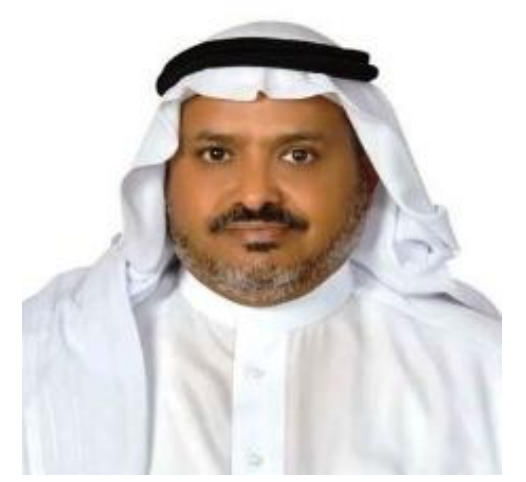

ABULLAH ALAMRI is a professor of earthquake seismology, Director of Seismic Studies Center at King Saud University (KSU). He is the President of the Saudi Society of Geosciences and editor-in-chief of the Arabian Journal of Geosciences (AJGS). He holds a B.S. in geology (1981) from King Saud University, M.Sc. (1985) in applied geophysics from University of South Florida, Tampa and Ph.D (1990) in earthquake seismology from University of Minnesota, USA. He is a member of Seismological Soc. of America, American Geophysical Union, European Ass. for Environmental \& Eng. Geophysics, Earthquakes Mitigation in the Eastern Mediterranean Region, National Comm. for Assessment \& Mitigation of Earthquake Hazards in Saudi Arabia, Mitigation of Natural Hazards Com at Civil Defense. His research interests are in the area of crustal structures and seismic micro zoning of the Arabian Peninsula. His recent projects involve also applications of EM and MT in deep groundwater exploration of Empty Quarter and geothermal prospecting of volcanic Harrats in the Arabian shield. He has published more than 150 research papers, achieved more than 45 research projects as well as authored several books and technical reports. He is a principal and Co-investigator in several national and international projects (KSU, KACST, NPST, IRIS, CTBTO, US Air force, NSF, UCSD, LLNL, OSU, PSU and Max Planck). He has also chaired and co-chaired several SSG, GSF, RELEMR workshops and forums in the Middle East. He obtained several worldwide prizes and awards for his scientific excellence and innovation. 
GR Focus Review

\title{
Pathways and challenges of the application of artificial intelligence to geohazards modelling
}

\author{
Abhirup Dikshit ${ }^{1}$, Biswajeet Pradhan ${ }^{1,2,3^{*}}$, Abdullah M. Alamri4 \\ ${ }^{1}$ Centre for Advanced Modelling and Geospatial Information Systems (CAMGIS), Faculty of \\ Engineering \& IT, University of Technology Sydney, Sydney, NSW 2007, Australia \\ ${ }^{2}$ Department of Energy and Mineral Resources Engineering, Sejong University, Choongmu- \\ gwan, 209, Neungdongro Gwangjin-gu, Seoul 05006, Korea \\ 3Center of Excellence for Climate Change Research, Department of Meteorology, King \\ Abdulaziz University, Jeddah 21589, Saudi Arabia \\ 4Department of Geology \& Geophysics, College of Science, King Saud University, Riyadh \\ 11451, Saudi Arabia
}

Corresponding Author: Biswajeet Pradhan, biswajeet.pradhan@uts.edu.au

\begin{abstract}
The application of artificial intelligence (AI) and machine learning in geohazard modelling has been rapidly growing in recent years, a trend that is observed in several research and application areas thanks to recent advances in AI. As a result, the increasing dependence on data driven studies has made its practical applications towards geohazards (landslides, debris flows, earthquakes, droughts, floods, glacier studies) an interesting prospect. These aforementioned geohazards were responsible for roughly $80 \%$ of the economic loss in the past two decades caused by all natural hazards. The present study analyses the various domains of geohazards which have benefited from classical machine learning approaches and highlights the future course of direction in this field. The emergence of deep learning has fulfilled several gaps in: i) classification; ii) seasonal forecasting as well as forecasting at longer lead times; iii) temporal based change detection. Apart from the usual challenges of dataset availability, climate change and anthropogenic activities, this review paper emphasizes that the future studies should focus on consecutive events along with integration of physical models. The recent catastrophe in Japan and Australia makes a compelling argument to focus towards consecutive events. The availability of higher temporal resolution and multi-hazard dataset will prove to be essential, but the key would be to integrate it with physical models which would improve our understanding of the mechanism involved both in single and consecutive hazard scenario. Geohazards would eventually be a data problem, like
\end{abstract}


geosciences, and therefore it is essential to develop models that would be capable of handling large voluminous data. The future works should also revolve towards interpretable models with the hope of providing a reasonable explanation of the results, thereby achieving the ultimate goal of Explainable AI.

Keywords: Machine learning; Deep learning; geo hazards; physical models; Explainable AI

\section{Introduction}

Artificial Intelligence (AI) is a key driver towards a fourth industrial revolution capable of boasting tremendous trans-disciplinary transformations. AI has made giant strides in various applications, from speech recognition (Hinton et al. 2012), self-driving vehicles (Farabet et al. 2012), computer vision (Krizhevsky et al. 2012) and natural language processing (Collobert et al. 2011), which was perceived as a task that could be performed only by humans (Hosny et al. 2018). Although, AI is very broad and there are several applications, in this paper, our discussion will be limited to geohazard studies related to "Machine Learning” and "Deep Learning”, which are often used interchangeably with AI and could led the differences among these to be unclear. Although, these terms are related to each other, but they are not same (Fig. 1). Machine learning is a technique of syntactic analysis of data, thereby learning from the data and finally make an informed decision (Alpaydin, 2014). On the other hand, deep learning is a subset of machine learning which is based on neural network structure and is considered being inspired by human brain (LeCun et al. 2015). For deep learning, the model extracts features itself whereas features are manually engineered in the case of classical machine learning (LeCun et al. 2015). While conducting the review, there seems to be quite an overlap of techniques which are almost inevitably described as machine learning, but are not actually machine learning. For e.g. - a lot of data mining approaches have been used and are usually referred as ML, but a key difference between these two exists. Data mining focusses on determining unknown properties in the data without the need of a specific goal, whereas ML needs a goal to perform a task based on known properties gathered from the training data (Buczak and Guven, 2016).

Although, past AI based techniques have led to subhuman performance results, the emergence of recent advanced deep learning algorithms have the capability to outperform human intelligence for specific problems (Mnih et al. 2015; Moravcík et al. 2017). The outperformance of deep learning methods in the game of Go is an accomplishment in itself, long considered being decades away from achieving such a feat given the complex nature of the board game involving an enormous number of potential moves (Silver et al. 2016). Researchers predict that AI has the capacity to outperform humans in several fields like 
language translation, writing books, transportation within the next decade (Grace et al. 2017).

Geohazards relate to geological, geomorphological, environmental or anthropogenic activities that have the potential to cause damage to human life, property and/or environment. The geohazard field itself is vast considering the development for different types, it is beyond the scope of this review, and therefore we focus on the six most disastrous geohazards, which are earthquakes, floods, droughts, landslides, debris flows and glacial lake outburst.

The use of AI in the field of geohazards has immense potential in several applications like prediction, change detection (Mezaal et al. 2018), early warning systems (Yang et al. 2019), vulnerability and risk management (Tehrany et al. 2015), spatial modelling (Rahmati et al. 2020) and mitigation measurement (Wang et al. 2019). The availability of vast amounts of remote sensing, climatic and atmospheric data has prompted researchers to identify new ways and techniques of making future reliable geohazards decision (Reichstein et al. 2019). Based on Scopus search, the use of highly advanced and complex algorithms is quite limited in this field when compared to computer science fields like image processing and natural language processing. The present article aims to analyse the current practices and suggest pathways in which the future research direction should proceed.

It is noteworthy to understand that still, most of the works analyse geohazard applications using machine learning techniques which are now slowly moving towards deep learning with few addressing intelligence quotient. In the subsequent sections, we highlight how the development of machine learning algorithms has improved our understanding in geohazard context, and highlight the use of deep learning for the major geohazards.

\section{Progress in Machine Learning Approaches}

The ML algorithms have the capability to learn the underlying system behaviour from a set of training data without the need of prior knowledge existing among the dataset (Schmidt et al. 2019). ML can be divided into three groups, supervised learning, unsupervised learning, and reinforcement learning (Jordan and Mitchell, 2015). Supervised learning attempts to determine an unknown function which correlates the input variables with an output variable, like classification and regression. Whereas, unsupervised learning only has input variable, and the model tries to discover patterns and structures, e.g. -clustering and association (Bishop, 2006). In between them is semi-supervised learning, where a large amount of input data is available but with only few output variables (Nguyen et al. 2017). Finally, reinforcement learning treats the problem of finding optimal or sufficiently good actions for a situation (Sutton and Barto, 2018). LeCun et al. (2015) in their path-breaking article on 
review of deep learning highlighted that unsupervised learning would eventually become more significant as human learning is largely unsupervised.

\subsection{ML in geohazard analysis}

The use of ML for various aspects of geohazard analysis is now pretty popular among researchers. This meteoritic rise in using ML techniques is primarily due to the availability of a vast amount of ground-based, climatic and remote sensing dataset (space-borne, airborne, terrestrial, etc.), along with the increase in computing ability over the past decade. It can be touted as an effective empirical approach for classification (supervised and unsupervised) and/or regression of non-linear systems (Lary, 2016). In the present study, we look into the application of ML in geohazard studies into four key areas: regression, classification, change detection and prediction.

The underlying assumption in almost every geohazard regression based study is that conditions responsible for occurrences would be the same in the future. Over the past decade, the use of regression techniques has focussed on spatial prediction, i.e., geohazard properties which are comparatively static during the analysed time period. These include geohazard studies related to aspects like susceptibility, vulnerability and risk. Here lies in the main challenge as some of the conditioning factors are inherently dynamic in nature and not static; like effects of climate change and effect of anthropogenic activities. Lately, these dynamics are being considered and regression techniques could help researchers to study such dynamics by analysing the temporal varying parameters. The classical problem faced by geohazard researchers is classification, of which ML models have gained enough traction and different geohazard aspects have been explored. Like, classifying a region into drought or non-drought areas or classifying into various degrees of susceptibility, hazard, vulnerability, and risk depending on the type of geohazard study being conducted. Further, the use of change anomaly detection techniques is key in almost every geohazard as it helps at two fronts, first being the identification of the historical hazard occurrences which could help in better understanding of the area (like, compiling landslide inventory and glacier outbursts) and second could aid in supporting the mitigation activities immediately (earthquake); or during (drought) the event. Both these aspects are crucial to understand the history of geohazards and providing relief works. But, arguably, the success story in any geohazard study is linked to how good can be the prediction capability, and whether we can predict it with greater accuracy and precision. What makes the field of geohazards so interesting and challenging at the same time as it not only depends on spatial and temporal context like in geosciences and earth sciences, but also on other aspects like the movement and volume estimation in case of landslides, glacier outbursts, or the propagation in case of droughts and 
floods. The further sections will look into which areas of various geohazards have benefitted the most out of ML and its implications on our understanding.

\subsubsection{Landslides and Debris Flows}

The most prominent use of ML for landslide study has been towards regression problems, especially understanding the susceptibility of an affected region. Susceptibility is defined as the likelihood of "where" a geohazard in an area can occur depending on local terrain conditions (Reichenbach et al. 2018; Dikshit et al. 2020a). These methods are primarily based on the analysis of geo-environmental variables which has caused such events historically, either using bivariate or multivariate statistics. The introduction of neural network showed better promising results with respect to logistic regression (Nefeslioglu et al. 2008), and thereafter a quest began to determine the best algorithm for a given study region. The studies have been based either on the use of a single approach like Support Vector Machine (SVM), Random Forest (RF), Artificial Neural Networks (ANN), Logistic Regression (Pradhan et al. 2010; Xu et al. 2012; Yariyan et al. 2020) or have used multiple ML models to find the best technique for a definite study region (Tien Bui et al. 2016; Chen et al. 2017). Landslide susceptibility which has seen the use of several models and found LR, ANN and RF to be most used (Felicísimo et al., 2013; Tien Bui et al. 2016; Chen et al. 2017). Huang and Zhao, (2018) conducted a review on the use of SVM models for landslide susceptibility purposes and suggested that the future studies should involve the use of hybrid models. Similar, has been the case with debris flow susceptibility, e.g.- Xiong et al. (2020) compared four different ML models (LR, SVM, RF and Boosted Regression Trees (BRT)) for Sichuan province in China and found BRT to be performing better. The reclassification of maps generated via regression techniques has also been studied using several ML techniques. The debris flow type classification saw the comparison among compared RF, AdaBoost, and Gradient Boosting (GBDT) and found RF to be the best performing (Wang et al. 2019). Another area where ML has made significant progress is extraction and classification (Ghorbanzadeh et al. 2019), which is carried out either at pixel or object level. The success in the use of ML has made researchers emphasize on improving its performance for both the aspects (Moosavi et al. 2014; Mezaal et al. 2018).

Lately, there also has been a focus on the study of dynamics of landslides, like analysing the movement and volume estimation. As an example, Krkač et al. (2017) used RF technique to predict the movement of landslides and found that it has the capability to predict the evolution of daily displacements for up to 30 days. Li et al. (2018) highlighted in their study that the use of classical ML algorithms may fail to understand landslide movement in case of heavy precipitation and suggested the use of extreme learning machine (ELM) approach. However, these approaches fail to capture the long temporal dependency for the input 
sequence, thereby the use of long short-term memory (LSTM) neural network was proposed by Yang et al. (2019). Shirzadi et al. (2017) compared various statistical and machine learning models to determine the landslide volume and found ANFIS model to be outperforming the other models. The displacement of landslide as a measure of forecasting was studied by Liu et al. (2014) wherein three different ML models were tested (SVM, Relevance Vector Machine and Gaussian Process) and found GP to be the best performing. These studies are encouraging and highlight the use of ML models to understand the dynamic components of landslides which would help overcome the dependence towards physical models.

\subsubsection{Droughts}

The aim to predict the occurrence of any geohazard in both spatial and temporal context has fascinated researchers for a long time, and drought prediction is probably the biggest challenge on both the fronts. Unlike other geohazards, where the onset is known, droughts do not have a clear definition of its onset and is depended on who is defining it (LloydHughes, 2014). The use of ANN has proved to be quite successful in drought prediction due to the non-linear behaviour (Mishra and Singh, 2010; Dikshit et al. 2020c). The major breakthrough in drought forecasting has been the use of lagged climatic variables as predictors, and has seen an immense improvement in forecasting results, especially for longer lead times (Hao et al. 2018). At present, drought prediction has seen the use of several basic and advanced ML models. Mishra and Singh, (2011); AghaKouchak et al. (2015) and Hao et al. (2018) in their review articles on drought prediction provide details about the various drought modelling approaches used in the literature. These articles emphasize that future works should look towards developing more advanced models and to assimilate data at higher temporal scale. The assimilation of data at higher temporal scales could help to unearth new prospects like forecasting flash droughts (Pendergrass et al. 2020). This would also help immensely in understanding drought propagation, i.e., how rainfall shortage disseminate to a deficit in low streamflow and soil moisture along with the influence of anthropogenic activities affecting this transformation (Van Loon et al. 2016). We have seen how improvement in human behaviour pushed the Zero Day in Cape Town in 2018 to an indefinite period (Simpkins, 2018). We believe that the accurate temporal determination of drought propagation can be achieved within the next decade, due to the availability of higher temporal resolution data from both remote sensing perspective (Himawari 8) and climate perspective (ERA5) along with improved time series forecasting models, particularly in deep learning context. The development of more advanced tools would help to forecast drought at longer lead times, possibly multi-years, surpassing the present limitations of 12 months. 
The study by Sutanto et al. (2019) proposed a novel methodology of using RF model to predict the impact of drought. For regression tasks, focus has more on the use of knowledgebased processes (e.g., Analytic Hierarchy Process) and the use of ML is fewer compared to prediction tasks. The study by Rahmati et al. (2020) exploited this aspect and compared six different ML models to analyse drought risk of south-east Australia.

\subsubsection{Earthquakes}

For long, there have been many apprehensions about the possibility of predicting earthquakes. Nagerestani et al. (2002) used Back Propagation Neural Network (BPNN) and Liu et al. (2004) utilising Radial Basis Function (RBF) neural networks to predict earthquakes. However, the failure of predicting the 2004 Parkfield earthquake (Bakun et al. 2005) was a setback as it involved massive resources both in terms of money and manpower. The introduction of advanced ML algorithms in the field has created a new hope among researchers to find patterns which could help in predicting earthquakes days ago in the foreseeable future. For this, ANN and RF have been used extensively, e.g. - Rouet-Leduc et al., (2017) used RF to predict the remaining time before the next failure derived from earthquakes, whereas Buscema et al. (2015) used ANN to estimate the magnitude of the events recorded daily and proving it to be a promising technique. Martínez-Álvarez et al. (2011) used regression to forecast to analyse the temporal patterns for an earthquake prediction. We expect that the biggest breakthrough in geohazards field would be an accurate prediction of earthquakes, primarily because of the scale of damage both in terms of lives and economy.

ML has also been successful to assess damage post-earthquake event, like Weiland et al. (2016) used SVM to detect such changes post March 2011 earthquake event in Japan. Comparing SVM with ANN reflected similar results while classifying damages post September 2018 event of Indonesia (Syifa et al. 2019). The regression problems like analysing seismic vulnerability which majorly depends on magnitude prediction along with structural and geological aspects have seen ANN as an effective model because the inherent nature of earthquake phenomena can be uncertain (Vicente et al. 2011; Jena et al. 2020a). The integration of vulnerability with hazard and probability to develop risk maps is essential to quantify the population at risk, which is often carried out using multi-criteria decision making and analytic hierarchy process (AHP) (Jena et al. 2020b). This is certainly an area where ML could prove to be immensely helpful as AHP is heavily dependent on the expert opinions and is subjective.

\subsubsection{Floods and glacier lake outbursts}


The prediction of floods has majorly initially involved the use of single algorithms like ANN, SVM, neuro-fuzzy, Multilayer Perceptron (MLP) which has slowly moved to the use of hybrid models like adaptive neuro-fuzzy inference systems (ANFIS), Wavelet Neural Network (WNN). ANNs have been mostly used and its performance among statistical models and/or other ML based models have been very well tested. The studies reveal that although ANN exceeds the performance compared to traditional statistical models, however, hybrid models have overcome the difficulties of the use of single ANN based models and achieved better accuracy. For flood susceptibility, RF model has proven to be successful over other conventional ML models (Chen et al. 2019; 2020) and several works have encouraged its use, especially when assessing at a national scale (Zhao et al. 2018). Tehrany et al. (2015) analysed the efficiency of SVM technique using four different kernel types for flood susceptibility at north-eastern part of Malaysia. Rahman et al. (2019) integrated statistical, ML and AHP to identify flood hazard areas in Bangladesh as an alternative to the conventional approaches (hydraulic and hydrological) that involves several parameters. More recently, Wagenaar et al. (2020) provided a comprehensive review of ML models in response to flood risk and impact assessment. Their findings suggest the possible use of ML under different conditions of exposure, hazard and impact. The study also predicts the areas where the use of ML is likely or unlikely to increase.

The change detection using ML has paved new ways in the field of glacier outbursts as it has been led to build an inventory for reliable Glacial Lake Outburst Flood (GLOF) hazard assessment. Veh et al. (2018; 2019) used RF model to develop such an inventory for Himalayan region. Dirsherl et al. (2020) used RF to classify glacier types in Antarctic ice sheet which is first of its kind study conducted on a large scale.

\subsection{Hybrid Models}

However, the domains studied under each geohazard is different from each other, with some avenues being more explored compared to other. As an example, landslide susceptibility mapping has been relatively more studied compared to other aspects like hazard and risk. The use of traditional machine learning has proved to be quite useful in understanding several traits of geohazard studies. However, analysing the complex nature involved with the increase in sample size and diversity has hampered the use of shallow learning algorithms due to their finite cell limit (Wang et al. 2019). The way forward to overcome this concern is by the integration of ML algorithms known as hybrid models or the use of deep learning. In almost all the various specific domains, the use of hybrid models have preceded the use of a single algorithmic approach. Hybrid models are basically ensembles developed by integrating algorithms that do not belong to the same family (Kadavi et al. 2018). Sameen et al. (2020) used integrated regularised greedy forests (RGF) and RF models for regression 
and classification purposes for Bhutan Himalayas. Fanos and Pradhan, (2019) used hybrid model to predict probable rockfall source in Malaysia and achieved accuracy of 0.93 . Alizadeh et al. (2018) used a hybrid framework involving ANN with Analytic Network Process (ANP) to assess earthquake vulnerability in north-western part of Iran. Zhou et al. (2016) forecasted slope displacement using Swarm Optimisation and SVM coupling approach, and the result showed an improvement in predictive abilities. Asencio-Cortés et al. (2018) used several regression algorithms along with stacking-based ensemble learning approach to predict earthquake magnitude. Such kind of study gives hope to ensemble learning, especially in the future for big data analytics. The use of hybrid models in case of flood studies have overcome the difficulties faced by the use of single ANN based models and achieved better accuracy. Like, the ability to predict short-term flood up to $2 \mathrm{hr}$ can be accurately done using single algorithms, but hybrid algorithms are better to predict longterm floods (Mosavi et al. 2018). Mostly, ANFIS has been used for hybrid algorithms for flood regression studies (Tien Bui et al. 2018). In general, it can be said that the use of hybrid models has proven to be more useful in terms of accuracy compared to single algorithmic use, however its use and application is dependent on the input data respective to geohazard type and the hybrid model being considered.

\section{Deep Learning}

Deep learning is basically a representation learning method composed of several representation layers, obtained by constituting non-linear modules. Each of these modules transforms the representation at every level which enables to learn complex functions (LeCun et al. 2015). The benefit of using such models is its ability to learn from the data instead of handcrafting features based on domain expertise. The field of deep learning is pretty new to geohazard researchers and there are several architectures, each having its own benefits and shortcomings. We highlight only the majorly used architectures, for an extensive understanding, readers are referred to Shrestha and Mahmood, (2019) and Ball et al. (2017) wherein a comprehensive review is provided. The application of deep learning types in geohazards has been on segmentation (Baumhoer et al. 2019), feature extraction (Ci et al. 2019), detection (Sameen and Pradhan, 2019) and prediction (Hu et al. 2019). The various types of image segmentation with their benefits and limitations have been elaborately discussed in Minaee et al. (2020).

\subsection{Deep learning architectures}

\subsubsection{Auto-encoder (AE)}

Auto-encoder (AE) can be considered as a special kind of ANN which can learn useful features from unsupervised data by reducing the reconstruction error between input and 
output values of the network (Bengio et al. 2007). It comprises encoder and decoder phases that could transform an input to representation and also has the ability to transform back to original signal, with the number of layers in output and output being similar and larger than hidden units (Fig. 2a). Hinton and Salakhutdinov, (2006) emphasized that such a design could reduce nonlinear dimensionality. In situations of high dimensional input data, single layer $\mathrm{AE}$ is ineffective and multiple layers are stacked to build Stacked $\mathrm{AE}$ (SAE) architecture. There are several variants of $\mathrm{AE}$ each serving a specific purpose like stacked denoising which intakes a corrupted sample data and are trained to recover the perfect "repaired" input to robustly obtain a good representation (Vincent et al. 2010).

\subsubsection{Deep belief network (DBN)}

The primary component of DBN are the Restricted Boltzmann machines (RBM) which involves one visible input and one hidden output layer, a type of generative model (Hinton and Sejnowski, 1986). The stacking of multiple RBMs builds DBN, wherein the output of a hidden layer of the preceding RBM is used as input of a visible layer for the following RBM (Hinton et al. 2006). The training of DBN is similar to SAE wherein the initial parameters are learned in a greedy layer-wise unsupervised pattern and thereby fine tuning the parameters using an additional layer at the top. The connections between visible and hidden inputs are restricted, with no connections between the nodes at same layer implying the visible units to be independent, which leads to efficient unsupervised training schemes (Hinton, 2002). This can be considered being more efficient compared to a single hidden layer using greedy layer-wise manner, as relatively better hierarchical representations can be learned which could better reflect the latent structures in the input data (Fig. 2b).

\subsubsection{Convolutional neural network (CNN)}

$\mathrm{CNN}$ network can be considered being inspired by human visual cortex with the capability to automatically capture and learn the spatial feature hierarchies and comprising three building blocks: convolutional, pooling and fully-connected layers (LeCun et al. 1998) (Fig. 2c). The first block is responsible to extract the local features from various locations of the raw input or capture the intervening features with learnable filters known as kernels. These kernels can also be optimised depending on the given problem with the aim to reduce gap between actual and output layers through an optimization algorithm like backpropagation and gradient descent. The pooling layer can be considered as a subsampling layer which reduces the size of the input layer and specifies either the maximum or average value from each subarea of the preceding layer. Finally, after completing an alternate stacking of convolutional and pooling layers, one or more fully-connected layers are added for classification or feature representation purposes. When multiple CNNs are stacked for capturing local geometric or 
spatial patterns, it is known as deep CNN. Some popular CNN architectures are AlexNet (Krizhevsky et al. 2012), GoogLeNet (Szegedy et al. 2015), VGG (Simonyan and Zisserman, 2015), ResNet (He et al. 2016).

\subsubsection{Recurrent neural network (RNN)}

The sole purpose of RNN which is a type of neural networks is to analyse non-stationary processes like time series data (Williams and Zipser, 1989). It can be considered as a series of interconnected networks for time series analysis and can be trained using back propagation based gradient descent algorithms. RNN considers both the current input data and the preceding data to map target vectors, a sharp contrast to neural networks which map target vectors by multiplying weights (Fig. 2d). One of the key advantages of using RNN is its ability to store an internal memory of previous inputs in the network, which enables it to recall key events that occurred several times in the past and could prove to be important in situations which are depended on past events. The issue arises when multiple RNNs are stacked, which leads to vanishing and exploding gradient problems (Bengio et al. 1994). This concern was tackled by the introduction of long short-term memory (LSTM) (Hochreiter and Schmidhuber, 1997). It consists of a cell which would store the values to be used at random intervals and three gates, viz., input, output and forget gate, to control and adjust the cell state.

With the increased interest towards the application of deep learning, various open source frameworks have also been developed. Figure 3 shows the most popular open source deep learning platforms based on the number of stars received in GitHub. The detailed discussion about each framework and its comparisons can be found at De Felice, (2017). Readers are also referred to https://developers.google.com/machine-learning/glossary/ and http://www.wildml.com/deep-learning-glossary/ for a complete glossary.

\subsection{Application of DL on geohazard studies}

The application to specific problems in geohazard are still in the early days, but few works have shown their capability to outperform ML and depicting promising results. The works of Ji et al. (2019) to identify damage buildings post 2010 Haiti earthquake using CNN architecture and its further improvement by $\mathrm{Ci}$ et al. (2019) to classify damage into various degrees, and not just damaged or not damaged are good signals for earthquake researchers. On the other hand, Mangalathu and Burton, (2019) used LSTM method to classify building damage based on textual descriptions of damage. The textual descriptions was based on crowd-sourced information gathered from citizens and social media platforms. Such kind of studies could prove to be effective in mitigation purposes as post any geohazard event. A barrage of information is available, which often makes it difficult to assess the credibility and 
the associated threat level. The use of deep learning has proved to be crucial for classification purposes in glacier fronts (Baumhoer et al. 2019) which used a modified U-net segmentation to classify calving fronts along Antarctic coastline. Such study is crucial as our understanding on glacial retreat needs to be improved as glacier melting due to climate change, and the subsequent rise in sea-level rise is an imminent threat in the future.

Similarly, in the case of landslide detection and mapping, researchers have attempted various architectures to improve classification. The use of CNN to identify landslides was attempted by Chen et al. (2018) wherein they analysed the changes under multi temporal images and utilized spatio-temporal context analysis. Lei et al. (2019) developed a CNN model with pyramid pooling instead of global pooling for developing a landslide inventory. Their model could explore the context of images efficiently and better detect landslides on various scales. Ghorbanzadeh et al. (2019) compared various conventional ML models with $\mathrm{CNN}$ and highlighted the efficacy of deep learning provided hyper parameters are carefully optimized. Sameen and Pradhan, (2019) trained a residual networks (ResNet) on spectral and topographic features, thereafter Prakash et al. (2020) used modified U-net segmentation along with ResNet to map landslide inventory.

The spatio-temporal prediction of floods was studied by $\mathrm{Hu}$ et al. (2019) wherein they developed a combination of novel LSTM model with Reduced Order Model (ROM) and the results were found to be complementary. The model was successfully able to analyse the spatio-temporal aspects, and such studies can help to predict floods assisting in near realtime predictions. For drought monitoring aspects, Shen et al. (2019) used a deep feedforward neural network for the prediction of Standardised Precipitation Evaporation Index (SPEI), a popular drought index at a regional scale in China and found a correlation with meteorological and agricultural droughts. The study by Wang et al. (2019) shows promising results on the use of $\mathrm{CNN}$ architecture for regression purposes for landslide susceptibility, which outperformed optimized SVM model. Pedro et al. (2018) attempted to detect earthquake by training labelled raw data with supervised CNNs to extract features that can efficiently differentiate seismic signals from seismic noise.

The use of DL in geohazard studies is still in infancy, with some prominent works in feature extraction and classification. The recent work by Pradhan et al. (2020) on the use of CNN for feature extraction and Word2Vec model to assign class attributes proved to be immensely helpful for change detection studies. Figure 4 highlights the various possible domains of geohazard studies and showing the trend in the possible studies towards achieving a datadriven environment. A more thorough understanding of other aspects like spatio-temporal modelling and improving predictive capability is bound to happen in the near future. 
One of the main differences between computer vision researchers and geohazard researchers is the vast difference in dataset availability and variants in classification. One way to understand it, is the ImageNet classification, which contains a database of images labelled by humans, like 'cat' or 'dog'. In such cases, there is a definitive answer to the labelling case, which may not true for geohazard community. For e.g. - labelling earthquake damages depending on the level of damage depends on a defined set of rules, which may or may not hold true for all parts of the world. Similarly, labelling a drought event can be tricky as it depends on the objectives of the study and the trends used to identify the drought. Also, usually it is difficult to find a set pattern among any recurring geohazards, like two drought events or earthquake events are not similar and most of the times the results determined need an external validation, from the premise which would provide the actual accuracy of the model. Also, images used in computer vision field typically have 3 bands (RGB), but hyperspectral images extend to hundreds of spectral channels well beyond the visible range, which often induce different statistical properties to those of natural images.

\section{What have we learnt?}

It can be well accepted that the use of highly advanced computational algorithms can solve several challenging aspects for every geohazard application. However, its use in earthquake and GLOF has been very minimal, therefore it's suggested that more algorithms needs to be tested in understanding aspects like movement and volume associated with GLOF or predictive capability of earthquakes. Also, the credibility of the results irrespective of the study conducted is highly dependent on the quality of the dataset and the predisposing factors being considered, which has been aptly pointed out in several studies (Dikshit et al. $2020 b)$. Therefore, in terms of data requirements, efforts should be made towards the use of higher spatial resolution and all the relevant causative factors. However, there may be still many parts of the world where higher resolution dataset may not be available, which has led several researchers to use the freely available Google Earth images.

The modelling aspects tell us that use of ML algorithms are well established, and the trend is more towards the use of hybrid models or comparing different single based ML algorithms along with ensemble techniques to determine the best modelling approach. This has been vastly used in terms of spatio-temporal modelling and regression techniques. Among the single based algorithmic approach, the most used models are RF, ANN, LR, SVM, decision trees and neuro-fuzzy. The use of better optimisation techniques has also been on a rise which could help in improving our understanding of the subject. One interesting aspect is the use of statistical tools (equal breaks, quantile, natural breaks) for reclassifying maps developed using various regression techniques, especially in the field of landslides and floods, thereby suggesting to use ML techniques for reclassifying maps. 
One of the major problems with ML is its ability to identify the subtleties due to the issue of insufficient data. This is where deep learning approaches can play an important role and is evident that it will surpass the accuracy obtained from ML based models in every aspect. The collection of enormous data to understand the correspondence between factors and the respective geohazard has been one of the concerns which researchers have tried to address. The use of either machine learning or deep learning approaches to study various areas of geohazards can be immensely complemented with the use of physical-based models. The use of physical-based models has their own challenges primarily owing to the in-depth knowledge and expertise of various parameters involved. The use of ML models in combination with physical models is one area which deserves more attention. The integration is required as the sole use of physical models has failed in several aspects. Generally, it has been used in tandem with statistical models (Goetz et al. 2011) and the focus should be on using ML models. Bellos and Tsakiris, (2016) coupled physical based 2D hydrodynamic model with hydrological unit graph theory which improved flood modelling for catchment area in Greece. This was further highlighted by Felder et al. (2017) which combined hydrodynamic and hydrological models which helped in improving the physical aspects of probable maximum flood.

\section{The future ahead}

There are several fronts which needs improvement for better understanding in every aspect of geohazards ranging from data availability, computational challenges, integration with physical models, application on a larger scale, interpretability of label features along with the ability to develop robust models to analyse multi hazard challenges. Some of the most pressing factors to be included in the future geohazard studies would be the effect of climate change and anthropogenic activities. The concerns about climate change and their influence on geohazard has been aptly highlighted in reviews discussing its effect on landslides (Gariano and Guzzetti, 2016), droughts (Ault, 2020), GLOF (Harrison et al. 2017). The causative factors in all the studied geohazards, except earthquakes, should include the factor of human activities, as it's increasingly becoming an important aspect (Gill and Malamud, 2017).

Here, we emphasize the need towards understanding consecutive geohazard events which are on a rise and will continue in the foreseeable future due to the ever changing climatic and anthropogenic landscape. Few recent examples of such events are the April 2015 Nepal earthquake, 2018 summer of Japan or the 2019 Australia summer. Zuccaro et al. (2018) describes a theoretical model for cascading effect scenario analysis and the basic framework towards identifying elements at risk. The Sendai Framework for Disaster Risk Reduction (UNDRR, 2015) and the Paris Agreement (UNFCCC, 2015) also calls for development 
towards formulating a comprehensive risk assessment framework. De Ruiter et al. (2020) attempted to fill this gap and provided the fundamental knowledge about multi-risk assessments. The general focus has been towards multi-hazard approaches which is a part of consecutive events and there are four key factors that needs to be considered while addressing consecutive events (Gill and Malamud, 2014): a) comparing various individual geohazards for a definite region b) considering all the various possible interactions between the geohazards c) impact of spatio-temporal interaction among hazards d) dynamic vulnerability and their effect on societal aspects.

Most of the studies have considered the first two factors and neglected the impacts of hazards which may coincide with spatially and/or temporally along with dynamic vulnerability aspects. The works on multi-hazard probability assessment is a small step towards our understanding of consecutive events and can be improved with the use of hybrid models or deep learning architecture. We recommend that researchers should emphasize towards improving the understanding of the possibility of such events and not solely focus on multi-hazard scenarios. The general scenario is analysing the coupling process, and the mechanism involved for a single geohazard event occurrence. There is an emergence towards collecting a vast pool of data at a higher temporal scale either based on sensor-based monitoring system or satellite imagery like Himawari 8 . The future would gradually move towards geostationary satellites images (Sawada et al. 2019) and/or climatic datasets like the ERA5 reanalysis data (C3S, 2017). The emergence of xBD dataset (Gupta et al. 2019) which includes 700,000 building damage labels corresponding to eight different disaster types is an encouraging step which can help in developing more novel methodologies. The focus for future studies should be on determining the possible scenarios after a hazard has occurred and this can be achieved by the amalgamation of data driven models and physical modelling approaches (Figure 5).

The trade-off between the prediction and explanation with DL models is a challenge with recent advances in interpretable models like post-hoc interpretability techniques (Rai, 2020). However, a consensus needs to be built on what domains are necessary for interpretability in geohazards. It's almost certain that prediction of an event needs a high level of explainability, but the accuracy in change anomaly topics while preparing inventory or regression techniques like sustainability is an open-ended question. We believe that studies such as damage assessment post a geohazard event, determining the temporal dynamics like landslide flow length or mapping flooded areas and segmentation problems need high accuracy and explainability should not be an issue.

\section{Conclusions and Future Recommendations}


The availability of large sums of data and our improved computational capability has made researchers focus towards the use of machine learning and subsequently deep learning. It is evident that some geohazard type (landslides, floods, drought) have benefitted more compared to other types (glacier outbursts). The challenges towards a better understanding of geohazards are enormous and therefore, we recommend the following:

1) The studies have focussed towards single hazards and therefore the focus should shift towards understanding consecutive event case scenarios using data-driven approaches. A step-by-step process should be followed towards this, wherein studies like comparison with ground-based data and remote sensing data should be analysed. There will be several key areas across the globe where either of the data collection methods would not give reliable results, which would make the optimisation techniques more significant.

2) The emergence of deep learning towards understanding the complex spatio-temporal relationships among variables is an encouraging step. However, its use across multi scales and sources are still limited which prevents it from using it frequently especially on a large scale (regional or national). These challenges are expected to be overcome in the near future with the availability of climatic dataset (ERA5), multi-hazard database (xBD) and higher temporal remote sensing data (Himawari 8). The future of geohazards could become a data problem similar to geosciences and therefore robust models need to be prepared which could aptly serve the purpose of understanding the mechanism involved behind a geohazard event.

3) Machine learning approaches use certain approximations which are considered being valid or are good approximations irrespective of the study being performed. Deep learning has the ability to overcome these issues and reckon that in future it will be the leading method for several geohazard related studies. It would also give an opportunity to researchers to tackle the issue of limited data either spatially or temporally, which can be very crucial for regions suffering from data availability.

4) Currently, even though ML or DL approaches help in spatio-temporal modelling, the physical relevance is not well-known. Few works have attempted to couple statistical models or used sensor-based instruments to understand the involved mechanism. However, the parameters used should work towards providing a sense of physical interpretation. The issue of uncertainty and extrapolation should be considered generating more confidence in the models used. The emergence of new datasets can help couple data driven models with physical models can help in a better understanding of the process. Such a process could eventually help in achieving explainable and interpretable ML models (Gunning, 2017), along with a combination of logic rules with deep neural networks (Reichstein et al. 2019). 
5) The evident change in climate and increase in anthropogenic activities would make consecutive events a usual norm in the future. Therefore, it becomes very significant working towards forecasting the possible geohazards after an event has occurred at different time scales (like possible events after few days, weeks or months) and can be very well complemented with physical models. This would also help in identifying the elements at risk, which could help in better mitigation strategies.

\section{Acknowledgements}

The study was financially supported by the Centre for Advanced Modelling and Geospatial Information Systems (CAMGIS), Faculty of Engineering and Information Technology, University of Technology Sydney (UTS). This research was also supported by Researchers Supporting Project number RSP-2020/14, King Saud University, Riyadh, Saudi Arabia. Authors would also like to thank the Professor M. Santosh for his editorial comments and anonymous reviewers for their careful review of the original manuscript and their valuable suggestions which helped us to improve the manuscript.

\section{References}

AghaKouchak, A., Farahmand, A., Melton, F., Teixeira, J., Anderson, M., Wardlow, B., Hain, C. (2015). Remote sensing of drought: Progress, challenges and opportunities. Reviews of Geophysics, 53, 452-480.

Alizadeh, M., Ngah, I., Hashim, M., Pradhan, B., Pour, A.B. (2018) A Hybrid Analytic Network Process and Artificial Neural Network (ANP-ANN) Model for Urban Earthquake Vulnerability Assessment. Remote Sens., 10, 975.

Asencio-Cortés G., Morales-Esteban A., Shang X., et al. (2018) Earthquake prediction in California using regression algorithms and cloud-based big data infrastructure. Comput. Geosci., 115, 198-210

Ault, T.B. (2020) On the essentials of drought in a changing climate. Science, 368 (6488), 256-260

Bakun, W., Aagaard, B., Dost, B. et al. (2005) Implications for prediction and hazard assessment from the 2004 Parkfield earthquake. Nature 437, 969-974

Ball, J. E., Anderson, D. T., Chan, C. S. (2017) Comprehensive survey of deep learning in remote sensing: theories, tools, and challenges for the community. J. Appl. Remote Sens. 11, 042609 
Barua, S., Ng, A., Perera, B. (2012) Artificial neural network-based drought forecasting using a nonlinear aggregated drought index. J. Hydrol. Eng., 17, 1408-1413

Baumhoer, C.A., Dietz, A.J., Kneisel, C., Kuenzer, C. (2019) Automated Extraction of Antarctic Glacier and Ice Shelf Fronts from Sentinel-1 Imagery Using Deep Learning. Remote Sens., 11, 2529.

Bellos, V., Tsakiris, G. (2016) A hybrid method for flood simulation in small catchments combining hydrodynamic and hydrological techniques. J. Hydrol., 540, 331-339.

Bengio, Y., Simard, P., Frasconi, P. (1994) Learning long-term dependencies with gradient descent is difficult. IEEE Trans. Neural Netw., 5, 157-166

Bengio, Y., Lamblin, P., Popovici, C., Larochelle, H. (2007) Greedy layer-wise training of deep networks. Advances in Neural Information Processing Systems, 153-160

Bishop, C. M. (2006) Pattern recognition and machine learning. Berlin: Springer.

Buczak, A.L., Guven, E. (2016) A Survey of Data Mining and Machine Learning Methods for Cyber Security Intrusion Detection. IEEE Commun. Surv. Tutor., 18 (2), 1153-1176

Chen, W., Xie, X., Wang, J., Pradhan, B. et al. (2017) A comparative study of logistic model tree, random forest, and classification and regression tree models for spatial prediction of landslide susceptibility. Catena, 151, 147-160

Chen, K.T., Prishchepov, A.V., Fensholt, R., Sabel, C.E. (2019) Detecting and monitoring long-term landslides in urbanized areas with nighttime light data and multi-seasonal Landsat imagery across Taiwan from 1998 to 2017. Remote Sens. Environ., 255, 317-327

Chen, W., Li, Y., Xue, W. et al. (2020) Modeling flood susceptibility using data-driven approaches of naïve Bayes tree, alternating decision tree, and random forest methods. Sci. Total Environ., 701, 134979

Ci, T., Liu, Z., Wang, Y. (2019) Assessment of the Degree of Building Damage Caused by Disaster Using Convolutional Neural Networks in Combination with Ordinal Regression. Remote Sens., 11, 2858

Collobert, R., et al. (2011) Natural language processing (almost) from scratch. J. Mach. Learn. Res. 12, 2493-2537.

Copernicus Climate Change Service (C3S) (2017) ERA5: Fifth generation of ECMWF atmospheric reanalyses of the global climate. Copernicus Climate Change Service Climate Data Store (CDS), https://cds.climate.copernicus.eu/cdsapp\#!/home 
Cooner, J.A., Shao, Y., Campbell, B.J. (2016) Detection of urban damage using remote Sens., 8, 868

Dang, V., Dieu, T.B., Tran, X. et al. (2019) Enhancing the accuracy of rainfall-induced landslide prediction along mountain roads with a GIS-based random forest classifier. Bull Eng Geol Environ 78, 2835-2849

De Felice, M. (2017) Which deep learning network is best for you? IDG Communications, [online] Available: http://www.cio.com/article/3193689/artificial-intelligence/which-deeplearning-network-is-best-for-you.html.

De Ruiter, M.C., Couasnon, A., van den Homberg, M.J.C. et al. (2020) Why We Can No Longer Ignore Consecutive Disasters. Earth's Future, 8, 3, e2019EFoo1425

Dikshit, A., Sarkar, R., Pradhan, B., et al. (2020a) Temporal Probability Assessment and Its Use in Landslide Susceptibility Mapping for Eastern Bhutan. Water 2020, 12, 267.

Dikshit, A., Sarkar, R., Pradhan, B., et al. (2020b) Rainfall Induced Landslide Studies in Indian Himalayan Region: A Critical Review. Appl. Sci., 10, 2466.

Dikshit, A., Pradhan, B., Alamri, A.M. (2020c) Short-Term Spatio-Temporal Drought Forecasting Using Random Forests Model at New South Wales, Australia. Appl. Sci., 10, 4254 .

Dirscherl, M., Dietz, A.J., Kneisel, C., Kuenzer, C. (2020) Automated Mapping of Antarctic Supraglacial Lakes Using a Machine Learning Approach. Remote Sens., 12, 1203.

Fanos, A.M., Pradhan, B. (2019) A Novel Hybrid Machine Learning-Based Model for Rockfall Source Identification in Presence of Other Landslide Types Using LiDAR and GIS. Earth Syst Environ 3, 491-506

Farabet, C., Couprie, C., Najman, L. \& LeCun, Y. (2012) Scene parsing with multiscale feature learning, purity trees, and optimal covers. In Proc. International Conference on Machine Learning http://arxiv.org/abs/1202.2160

Feldar, G., Zischg, A., Weingartner, R. (2017) The effect of coupling hydrologic and hydrodynamic models on probable maximum flood estimation. J. Hydrol., 550, 157-165

Felicísimo, Á.M., Cuartero, A., Remondo, J. et al. (2013) Mapping landslide susceptibility with logistic regression, multiple adaptive regression splines, classification and regression trees, and maximum entropy methods: a comparative study. Landslides 10, 175-189 
Gariano, S.L., Guzzetti, F. (2016) Landslides in a changing climate. Earth-Science Rev., 162, 227-252

Ghorbanzadeh, O., Blaschke, T., Gholamnia, K., Meena, S.R., Tiede, D., Aryal, J. (2019) Evaluation of Different Machine Learning Methods and Deep-Learning Convolutional Neural Networks for Landslide Detection. Remote Sens., 11, 196.

Gill, J.C., Malamud, B.D. (2014) Reviewing and visualizing the interactions of natural hazards. Reviews of Geophysics, 52, 680-722

Gill, J.C., Malamud, B.D. (2017) Anthropogenic processes, natural hazards, and interactions in a multi-hazard framework. Earth-Science Reviews, 166, 246-269

Goetz, J.N., Guthrie, R.H., Brenning, A. (2011) Integrating physical and empirical landslide susceptibility models using generalized additive models. Geomorphology 129:376-386.

Grace, K., Salvatier, J., Dafoe, A., Zhang, B. \& Evans, O. (2017) When will AI exceed human performance? Evidence from AI experts. arXiv Preprint arXiv:1705.08807

Gunning, D. (2017) Explainable Artificial Intelligence (XAI)

Gupta, R., Goodman, B., Patel, N., et al. (2019) Creating xbd: A dataset for assessing building damage from satellite imagery. In Proceedings of the CVPR Workshops, Long Beach, CA, USA, 16-20 June 2019

Hao, Z., Singh, V.P., Xia, Y. (2018) Seasonal Drought Prediction: Advances, Challenges, and Future Prospects. Reviews of Geophysics, 56(1):108-141

Harrison, S., Kargel, J.S., Huggel, C. et al. (2017) Climate change and the global pattern of moraine-dammed glacial lake outburst floods. The Cryosphere, 12, 1195-1209

He, K., Zhang, X., Ren, S., Sun, J. (2016) Deep residual learning for image recognition. Proceedings of the IEEE Conference on Computer Vision and Pattern Recognition, 770-778

Hinton, G.E., Sejnowski, T.J. (1986) Learning and Relearning in Boltzmann Machines. Parallel Distributed Processing: Explorations in the Microstructure of Cognition 1, 282-317

Hinton, G.E. (2002) Training products of experts by minimizing contrastive divergence. Neural Comput., 14, 1771-1800

Hinton, G.E., Salakhutdinov, R.R. (2006) Reducing the dimensionality of data with neural networks. Science, 313, 504-507

Hinton, G.E., Osindero, S., Teh, Y.W. (2006) A fast learning algorithm for deep belief nets. Neural Comput., 18, 1527-1554 
Hochreiter, S., Schmidhuber, J. (1997) Long short-term memory. Neural Comput., 9, 17351780

Hosny, A., Parmar, C., Quackenbush, J. et al. (2018) Artificial intelligence in radiology. Nat Rev Cancer 18, 500-510

Hu, R., Fang, F., Pain, C.C., Navon, I.M. (2019) Rapid spatio-temporal flood prediction and uncertainty quantification using a deep learning method. J. Hydrol. 575, 911-920

Huang, Y., Zhao, L. (2018) Review on landslide susceptibility mapping using support vector machines. Catena, 165, 520-529

Jena, R., Pradhan, B., Beydoun, G. (2020a) Earthquake vulnerability assessment in Northern Sumatra province by using a multi-criteria decision-making model. Int. J. Disaster Risk Reduct., 46, 101518

Jena, R., Pradhan, B., Beydoun, G. et al. (2020b) Integrated model for earthquake risk assessment using neural network and analytic hierarchy process: Aceh province, Indonesia. Geoscience Frontiers, 11(2), 613-634

Ji, M., Liu, L., Du, R., Buchroithner, M.F. (2019) A Comparative Study of Texture and Convolutional Neural Network Features for Detecting Collapsed Buildings After Earthquakes Using Pre- and Post-Event Satellite Imagery. Remote Sens., 11, 1202

Jordan, M.I., Mitchell, T.M. (2015) Machine learning: trends, perspectives, and prospects. Science, 349, 255-260

Kadavi, P., Lee, C.W., Lee, S. (2018) Application of ensemble-based machine learning models to landslide susceptibility mapping. Remote Sensing, 10, 1252

Krizhevsky, A., Sutskever, I. \& Hinton, G. (2012) ImageNet classification with deep convolutional neural networks. In Proc. Advances in Neural Information Processing Systems 25 1090-1098.

Krkač, M., Špoljarić, D., Bernat, S. et al. (2017) Method for prediction of landslide movements based on random forests. Landslides 14, 947-960

Lary, D.J., Alavi, A.H., Gandomi, A.H., Walker, A.L. (2016) Machine learning in geosciences and remote sensing. Geoscience Frontiers, 7(1):3-10

LeCun, Y., Bengio, Y., Hinton, G. (2015) Deep learning. Nature 521, 436-444

LeCun, Y., Bottou, L., Bengio, Y., Haffner, P. (1998) Gradient-based learning applied to document recognition. Proc. IEEE, 86, 2278-2324 
Lei, T., Zhang, Y., Lv, Z. et al. (2019) Landslide inventory mapping from bitemporal images using deep convolutional neural networks. IEEE Geosci. Remote Sens. Lett., 16, 6, 982-986

Liu, Z., Shao, J., Xu, W. et al. (2014) Comparison on landslide nonlinear displacement analysis and prediction with computational intelligence approaches. Landslides 11, 889-896 Lloyd- Hughes B. (2014) The impracticality of a universal drought definition. Theor Appl Climatol, 117:607-611

Mangalathu, S., Hwang, S.H., Choi, E. et al. (2019) Rapid seismic damage evaluation of bridge portfolios using machine learning techniques. Engineering Structures, 201, 109785

Mangalathu, S., Burton, H.V. (2019) Deep learning-based classification of earthquakeimpacted buildings using textual damage descriptions. Int. J Disaster Risk Reduction, 36, 101111

Mangalathu, S., Sun, H., Nweke, C.C., Yi, Z., Burton, H.V. (2020) Classifying earthquake damage to buildings using machine learning. Earthq. Spectra, 36(1), 183-208

Martínez-Álvarez F., Troncoso A., Morales-Esteban A., Riquelme J.C. (2011) Computational Intelligence Techniques for Predicting Earthquakes. In: Corchado E., Kurzyński M., Woźniak M. (eds) Hybrid Artificial Intelligent Systems. HAIS 2011. Lecture Notes in Computer Science, vol 6679. Springer, Berlin, Heidelberg

Mezaal, M., Pradhan, B., Rizeei, H. (2018) Improving landslide detection from airborne laser scanning data using optimized Dempster-Shafer. Remote Sens., 10, 1029.

Minaee, S., Boykov, Y., Porikli, F., et al. (2020) Image Segmentation Using Deep Learning: A Survey. https://arxiv.org/abs/2001.05566

Mishra A., Desai, V., Singh, V. (2007) Drought forecasting using a hybrid stochastic and neural network model. J. Hydrol. Eng., 12,626-638

Mishra, A. K., Singh, V. P. (2011) Drought modelling - A review. J. Hydrol., 403(1-2), 157175

Mnih, V., Kavukcuoglu, K., Silver, D. et al. (2015) Human-level control through deep reinforcement learning. Nature 518, 529-53

Moosavi, V., Talebi, A., Shirmohammadi, B. (2014) Producing a landslide inventory map using pixel-based and object-oriented approaches optimized by Taguchi method. Geomorphology, 204, 646-656 
Mosavi, A., Ozturk, P., Chau, K.-W. (2018) Flood Prediction Using Machine Learning Models: Literature Review. Water, 10, 1536.

Moravc``́k, M. et al. (2017) DeepStack: expert-level artificial intelligence in heads-up nolimit poker. Science 356, 508-513

Nefeslioglu, H.A., Gokceoglu, C., Sonmez, H. (2008) An assessment on the use of logistic regression and artificial neural networks with different sampling strategies for the preparation of landslide susceptibility maps. Engineering Geology, 97, 171-191

Pendergrass, A.G., Meehl, G.A., Pulwarty, R. et al. (2020) Flash droughts present a new challenge for subseasonal-to-seasonal prediction. Nat. Clim. Chang. 10, 191-19

Perol, T., Gharbi, M., Denolle, M. (2018) Convolutional neural network for earthquake detection and location. Sci. Adv., 4, e1700578

Pradhan, B., Lee, S. (2010) Landslide susceptibility assessment and factor effect analysis: backpropagation artificial neural networks and their comparison with frequency ratio and bivariate logistic regression modelling. Environment Modelling \& Software, 25(6):747-759

Pradhan, B., Al-Najjar, H.A.H., Sameen, M.I. et al. (2020) Unseen Land Cover Classification from High-Resolution Orthophotos Using Integration of Zero-Shot Learning and Convolutional Neural Networks. Remote Sens., 12, 1676.

Prakash, N., Manconi, A., Leow, S. (2020) Mapping Landslides on EO Data: Performance of Deep Learning Models vs. Traditional Machine Learning Models. Remote Sensing, 12, 346

Rahman, M., Ningsheng, C., Islam, M.M. et al. (2019) Flood Susceptibility Assessment in Bangladesh Using Machine Learning and Multi-criteria Decision Analysis. Earth Syst Environ 3, 585-601

Rahmati O., Falah, F., Dayal, K.S. et al. (2020) Machine learning approaches for spatial modeling of agricultural droughts in the south-east region of Queensland Australia. Sci. Total Environ., 699, 134230

Rai, A. (2020) Explainable AI: from black box to glass box. J. of the Acad. Mark. Sci. 48, $137-141$

Reichenbach, P., Rossi, M., Malamud, B.D., Mihir, M., Guzzetti, F. (2018) A review of statistically-based landslide susceptibility models. Earth-Sci. Rev., 180, 60-91

Reichstein, M., Camps-Valls, G., Stevens, B. et al. (2019) Deep learning and process understanding for data-driven Earth system science. Nature 566, 195-204 
Sameen, M.I. Pradhan, B. (2019) Landslide Detection Using Residual Networks and the unsupervised factor optimisation and regularised greedy forests. Computers \& Geosciences, 134,104336

Sawada, Y., Okamoto, K., Kunii, M., Miyoshi, T. (2019) Assimilating every-10-minute Himawari-8 infrared radiances to improve convective predictability. J. Geophys. Res. Atmos., 124, 2546-2561

Shirzadi, A., Shahabi, H., Chapi, K., et al. (2017) A comparative study between popular statistical and machine learning methods for simulating volume of landslides. Catena 157:213-226

Shen, R., Huang, A., Li, B., Guo, J. (2019) Construction of a drought monitoring model using deep learning based on multi-source remote sensing data. Int. J. Appl. Earth Obs. Geoinformation 79, 48-57

Shrestha, A. Mahmood, A., (2019) Review of deep learning algorithms and architectures, IEEE Access, 7, 53040-53065

Silver, D. et al. (2016) Mastering the game of Go with deep neural networks and tree search. Nature 529, 484-489

Simonyan, K., Zisserman, A. (2015) Very deep convolutional networks for large-scale image recognition. Proceedings of the ICLR

Simpkins, G. (2018) Running dry. Nature Clim Change 8, 369

Sutanto, S.J., van der Weert, M., Wanders, N. et al. (2019) Moving from drought hazard to impact forecasts. Nat Commun 10, 4945.

Sutton, R.S., Barto, A.G. (2018) Reinforcement learning: An introduction. MIT Press, Cambridge, MA

Syifa, M., Kadavi, P.R., Lee, C.-W. (2019) An Artificial Intelligence Application for PostEarthquake Damage Mapping in Palu, Central Sulawesi, Indonesia. Sensors, 19, 542

Szegedy, C., Liu, W., Jia, Y. et al. (2015) Going deeper with convolutions Proceedings of the IEEE Conference on Computer Vision and Pattern Recognition, 1-9

Tien Bui, D., Tuan, T.A., Klempe, H., Pradhan, B., Revhang, I (2016) Spatial prediction models for shallow landslide hazards: a comparative assessment of the efficacy of support 
vector machines, artificial neural networks, kernel logistic regression, and logistic model tree. Landslides 13, 361-378

Tien Bui, D., Khosravi, K., Li, S., et al. (2018) New Hybrids of ANFIS with Several Optimization Algorithms for Flood Susceptibility Modeling. Water, 10, 1210.

Tehrany, M.S., Pradhan, B., Mansoor, S., Ahmad, N. (2015) Flood susceptibility assessment using GIS-based support vector machine model with different kernel types. Catena, 125, 91101

UNDRR (2015). Sendai framework for disaster risk reduction 2015-2030. Retrieved from https://www.unisdr.org/files/43291 sendaiframeworkfordrren.pdf

UNFCCC (2015) Adoption of the Paris Agreement. Report No. FCCC/CP/2015/L.9/Rev.1, http://unfccc.int/resource/docs/2015/cop21/eng/logro1.pdf

Van Loon, A.F. et al. (2016) Drought in the Anthropocene. Nat. Geosci., 9, 89-91

Veh, G., Korup, O., Roessner, S., Walz, A. (2018) Detecting Himalayan glacial lake outburst floods from Landsat time series. Remote Sens. Environ., 207, 84-97

Veh, G., Korup, O., von Specht, S. et al. (2019) Unchanged frequency of moraine-dammed glacial lake outburst floods in the Himalaya. Nat. Clim. Chang. 9, 379-383

Vicente, R., Parodi, S., Lagomarsino, S., Varum, H., Silva, J.M. (2011) Seismic vulnerability and risk assessment: Case study of the historic city centre of Coimbra, Portugal. Bull. Earthq. Eng., 9, 1067-1096

Vincent, P., Larochelle, H., Lajoie, I. et al. (2010) Stacked denoising autoencoders: learning useful representations in a deep network with a local denoising criterion. J. Mach. Learn. Res., 11, 3371-3408

Wagenaar, D., Curran, A., Balbi, M., et al. (2020) Invited perspectives: How machine learning will change flood risk and impact assessment, Nat. Hazards Earth Syst. Sci., 20, $1149-1161$

Wang, N., Cheng, W., Zhao, M. et al. (2019) Identification of the Debris Flow Process Types within Catchments of Beijing Mountainous Area. Water, 11, 638.

Wang, Y., Fang, Z., Hong, H. (2019) Comparison of convolutional neural networks for landslide susceptibility mapping in Yanshan County, China. Sci. Total Environ., 666, 975993

Wang, H., Zhang, L., Yin, K. et al. (2020) Landslide identification using machine learning. https://doi.org/10.1016/j.gsf.2020.02.012 
Wieland, M., Liu, W., Yamazaki, F. (2016) Learning Change from Synthetic Aperture Radar Tsunami-Induced Changes. Remote Sens., 8, 792

Williams, R.J., Zipser, D. (1989) A learning algorithm for continually running fully recurrent neural networks. Neural Comput., 1, 270-280

Xiong, K., Adhikari, B.R., Stamatopoulos, C.A., et al. (2020) Comparison of Different Machine Learning Methods for Debris Flow Susceptibility Mapping: A Case Study in the Sichuan Province, China. Remote Sens., 12, 29

Xu, C., Dai, F., Xu, X., Lee, Y.H. (2012) GIS-based support vector machine modeling of earthquake-triggered landslide susceptibility in the Jianjiang River watershed, China. Geomorphology, 145-146, 70-80

Yang, B., Yin, K., Lacasse, S. et al. (2019) Time series analysis and long short-term memory neural network to predict landslide displacement. Landslides 16, 677-694

Yariyan, P., Avand, M., Soltani, F., et al. (2020) Earthquake Vulnerability Mapping Using Different Hybrid Models. Symmetry, 12, 405.

Zhao, G., Pang, B., Xu, Z. et al. (2018) Mapping flood susceptibility in mountainous areas on a national scale in China. Sci. Total Environ., 615, 1133-1142

Zhou, C., Yin, K., Cao, Y., Ahmed, B. (2016) Application of time series analysis and PSOSVM model in predicting the Bazimen landslide in the Three Gorges Reservoir, China. Engineering Geology, 204, 108-120

Zuccaro, G., De Gregorio, D., Leone, M. (2018) Theoretical model for cascading effects analyses. Int. Journal of Disaster Risk Reduction, 30, 199-215 


\section{Figure captions}

Figure 1: Various aspects of AI.

Figure 2: Various Deep Learning architectures: (a) Auto-encoder, (b) Deep belief network, (c) Convolutional neural networks, and (d) Recurrent neural networks.

Figure 3: Popular deep learning frameworks based on GitHub count as of April, 2020.

Figure 4: Flowchart highlighting the various aspects of geohazard studies and the current focus towards models used along with the future scenario.

Figure 5: Flowchart highlighting the current practices and the future aspects expected in order to achieve better intelligence quotient and gradual understanding towards understanding of the mechanism and interpretability providing "Explainable AI".

\section{Table captions}

Table 1: Strengths and limitations of various deep learning methods in various geohazard studies. 


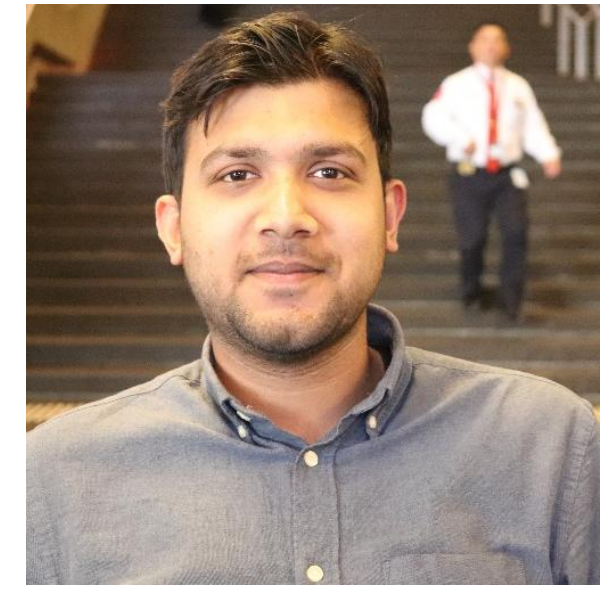

ABHIRUP DIKSHIT is a PhD student at the Centre for Advanced Modelling and Geospatial Information Systems (CAMGIS), University of Technology, Sydney. He has completed his Master (MS by Research) in Civil Engineering from the International Institute of Information Technology (IIIT) Hyderabad, India. He was awarded the International Research Training Program Scholarship (IRTP) by the Australian Government to pursue his doctoral research.

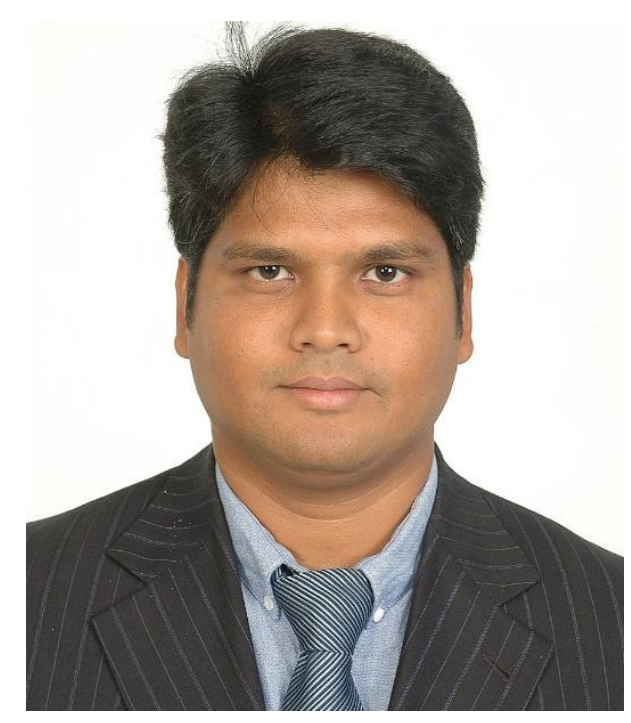

BISWAJEET PRADHAN received the Habilitation in remote sensing from the Dresden University of Technology, Germany, in 2011. He is founding Director of the Centre for Advanced Modelling and Geospatial Information Systems (CAMGIS), Faculty of Engineering and IT. He is also the Distinguished Professor with the University of Technology Sydney. He is also an internationally established Scientist in the fields of geospatial information systems (GIS), remote sensing and image processing, complex modeling/geo-computing, machine learning and soft-computing applications, natural hazards, and environmental modeling. Since 2015, he has been serving as the Ambassador Scientist for the Alexander Humboldt Foundation, Germany. Out of his more than 450 articles, more than 400 have been published in science citation index (SCI/SCIE) technical journals. He has authored eight books and 13 book chapters. He was a recipient of the Alexander von Humboldt Fellowship from Germany. He received 55 awards in recognition of his excellence in teaching, service, and research, since 2006. He was also a recipient of the Alexander von Humboldt Research 
Fellowship from Germany. From 2016 to 2019, he was listed as the World's Most Highly Cited Researcher by Clarivate Analytics Report as one of the world's most influential mind. In 2018 and 2019, he was awarded as the World Class Professor by the Ministry of Research, Technology and Higher Education, Indonesia. He is also an Associate Editor and an Editorial Member of more than eight ISI journals. He has widely travelled abroad, visiting more than 55 countries to present his research findings.

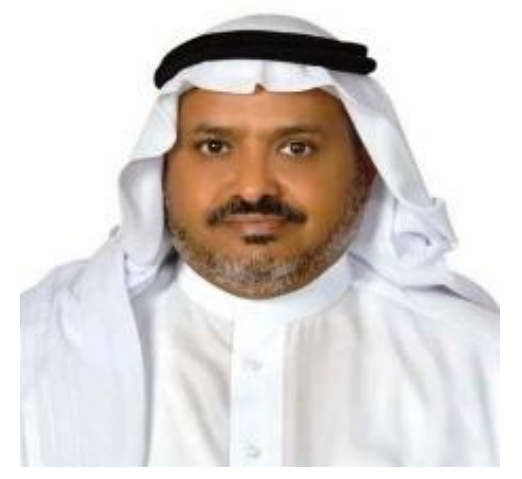

ABULLAH ALAMRI, M.S.; is a professor of earthquake seismology, Director of Seismic Studies Center at King Saud University (KSU). He is the President of the Saudi Society of Geosciences and editor-in-chief of the Arabian Journal of Geosciences (AJGS). He holds a B.S. in geology (1981) from King Saud University, M.Sc. (1985) in applied geophysics from University of South Florida, Tampa and Ph.D (1990) in earthquake seismology from University of Minnesota, USA. He is a member of Seismological Soc. of America, American Geophysical Union, European Ass. for Environmental \& Eng. Geophysics, Earthquakes Mitigation in the Eastern Mediterranean Region, National Comm. for Assessment \& Mitigation of Earthquake Hazards in Saudi Arabia, Mitigation of Natural Hazards Com at Civil Defense. His research interests are in the area of crustal structures and seismic micro zoning of the Arabian Peninsula. His recent projects involve also applications of EM and MT in deep groundwater exploration of Empty Quarter and geothermal prospecting of volcanic Harrats in the Arabian shield. He has published more than 150 research papers, achieved more than 45 research projects as well as authored several books and technical reports. He is a principal and Co-investigator in several national and international projects (KSU, KACST, NPST, IRIS, CTBTO, US Air force, NSF, UCSD, LLNL, OSU, PSU and Max Planck). He has also chaired and co-chaired several SSG, GSF, RELEMR workshops and forums in the Middle East. He obtained several worldwide prizes and awards for his scientific excellence and innovation. 
Click here to download high resolution image

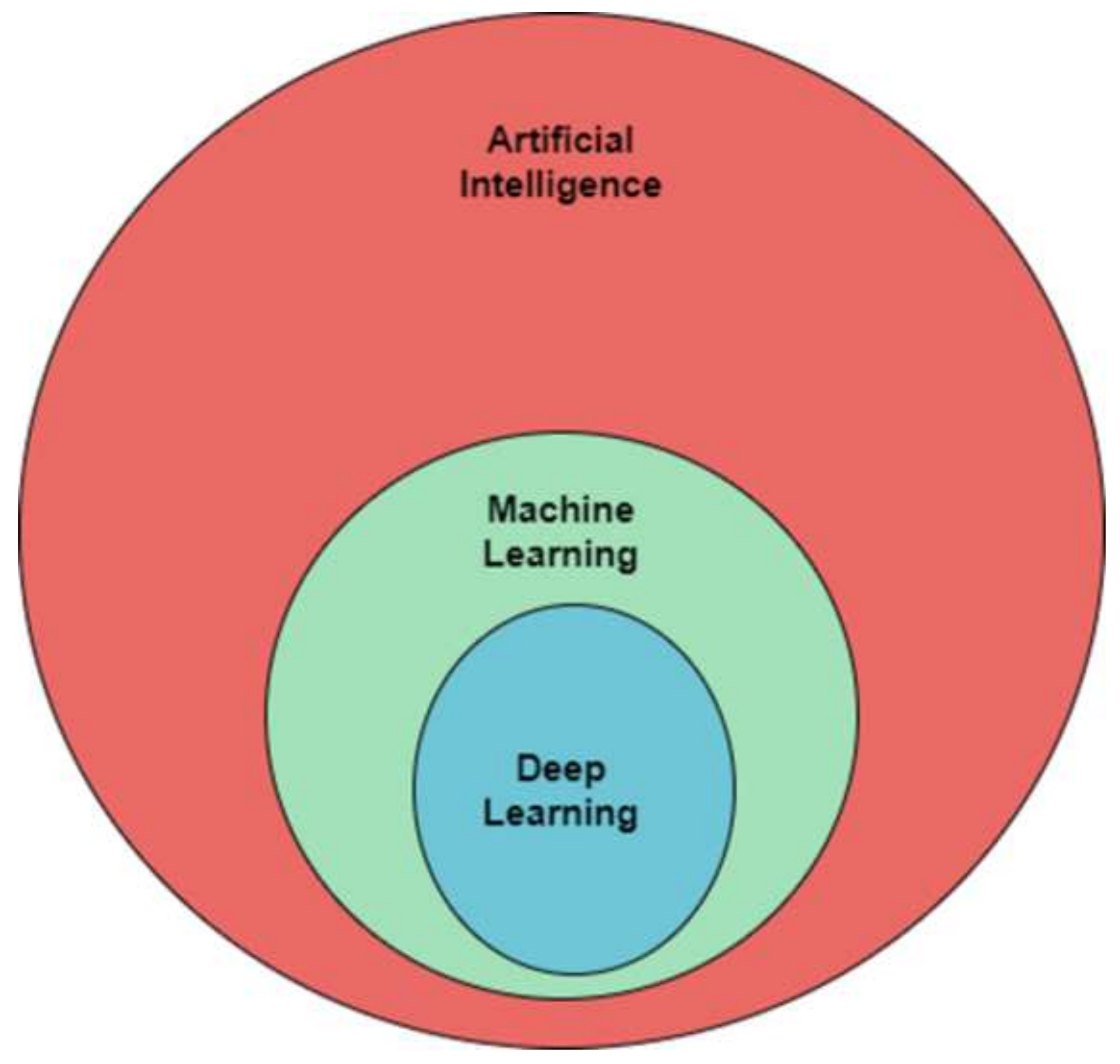




\section{Figure 2}

Click here to download high resolution image

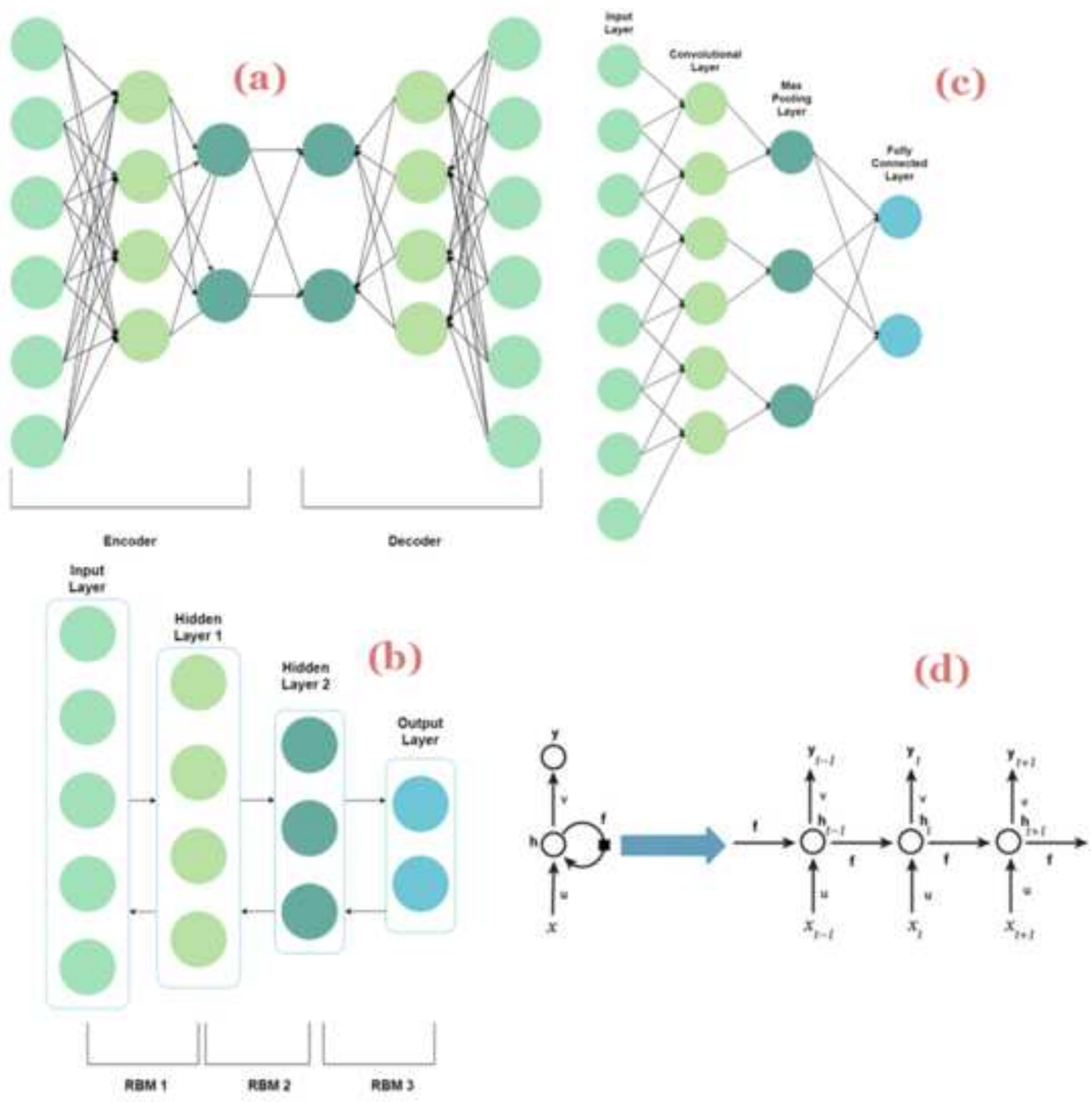




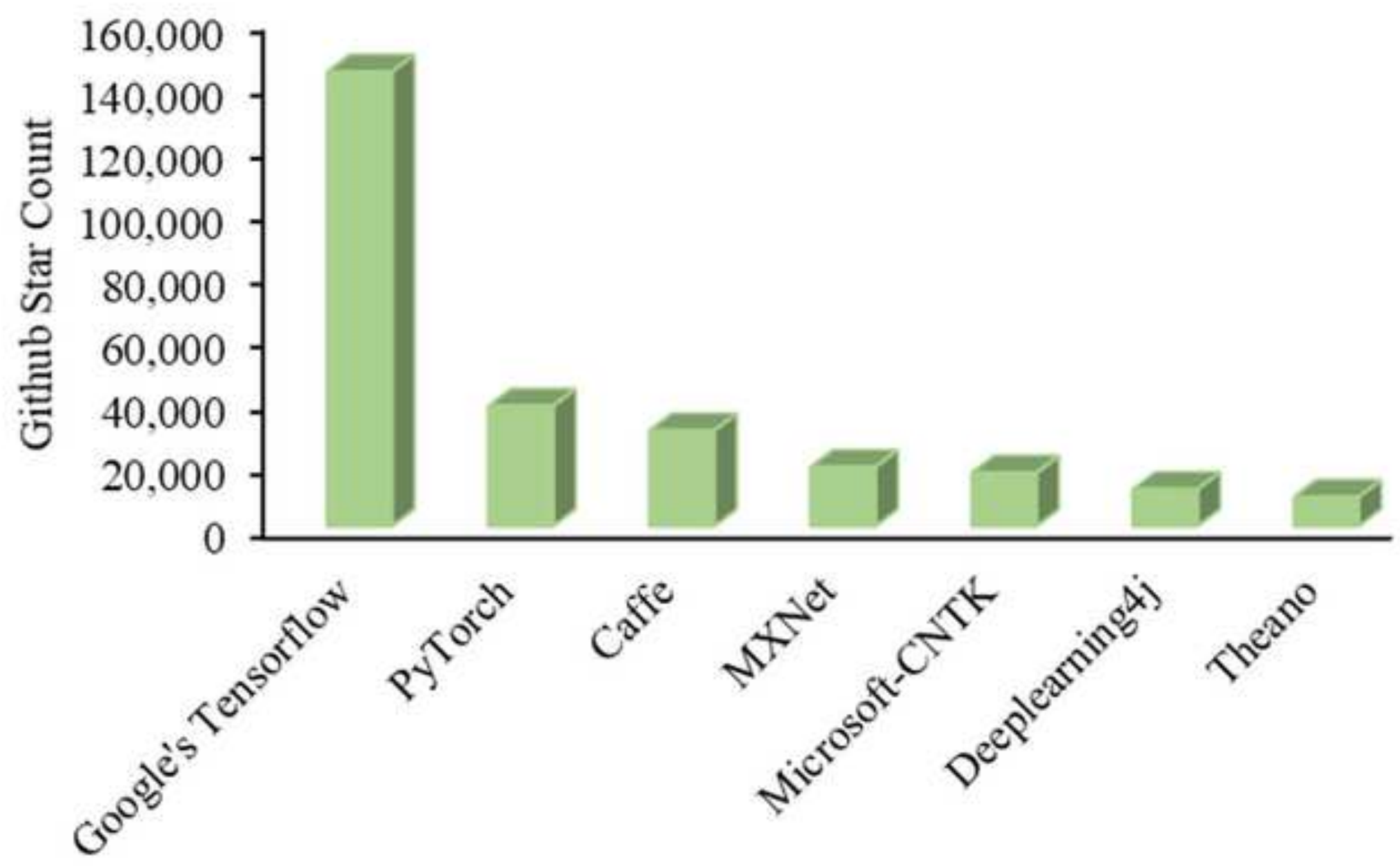


Click here to download high resolution image

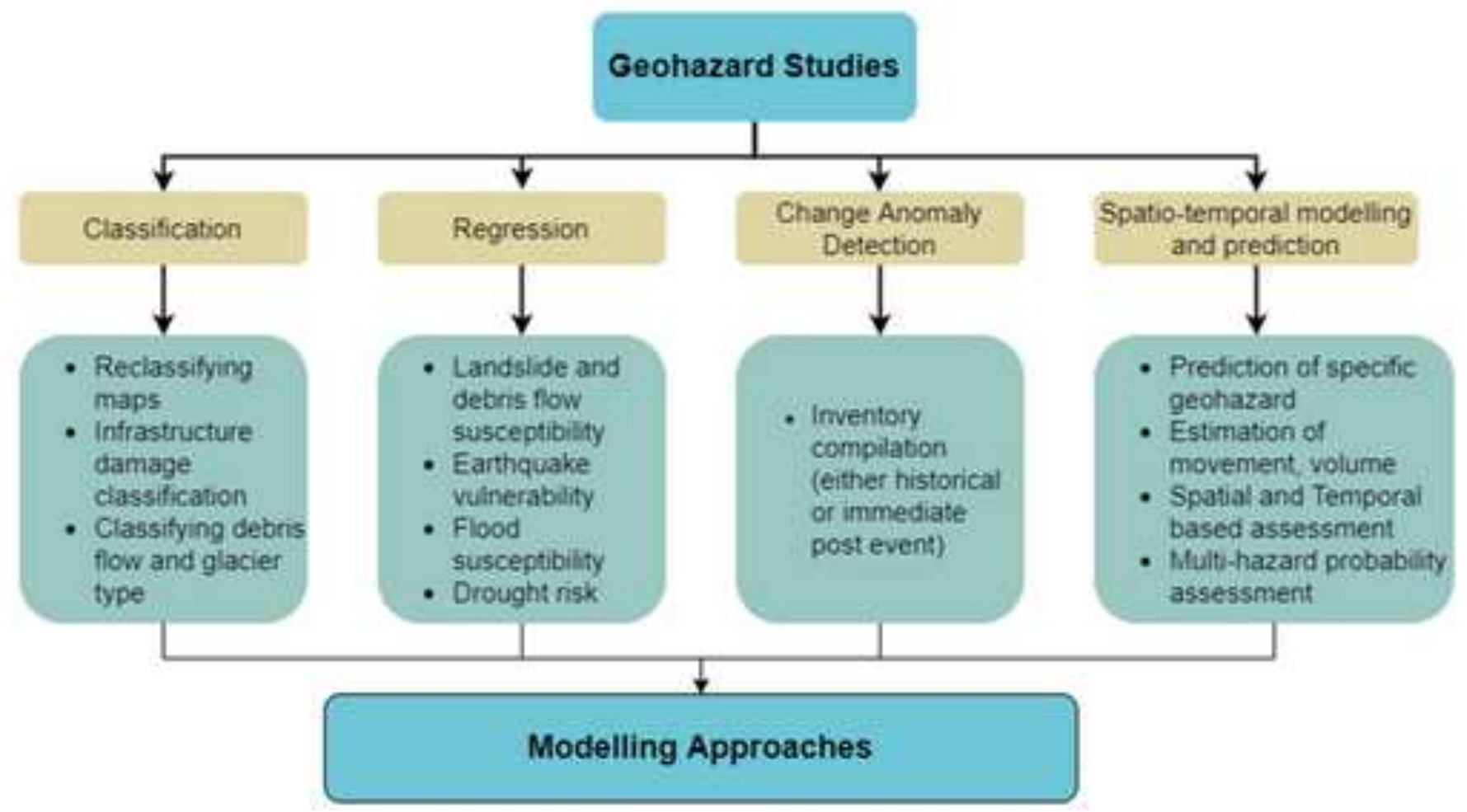

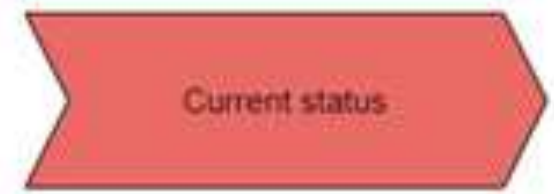
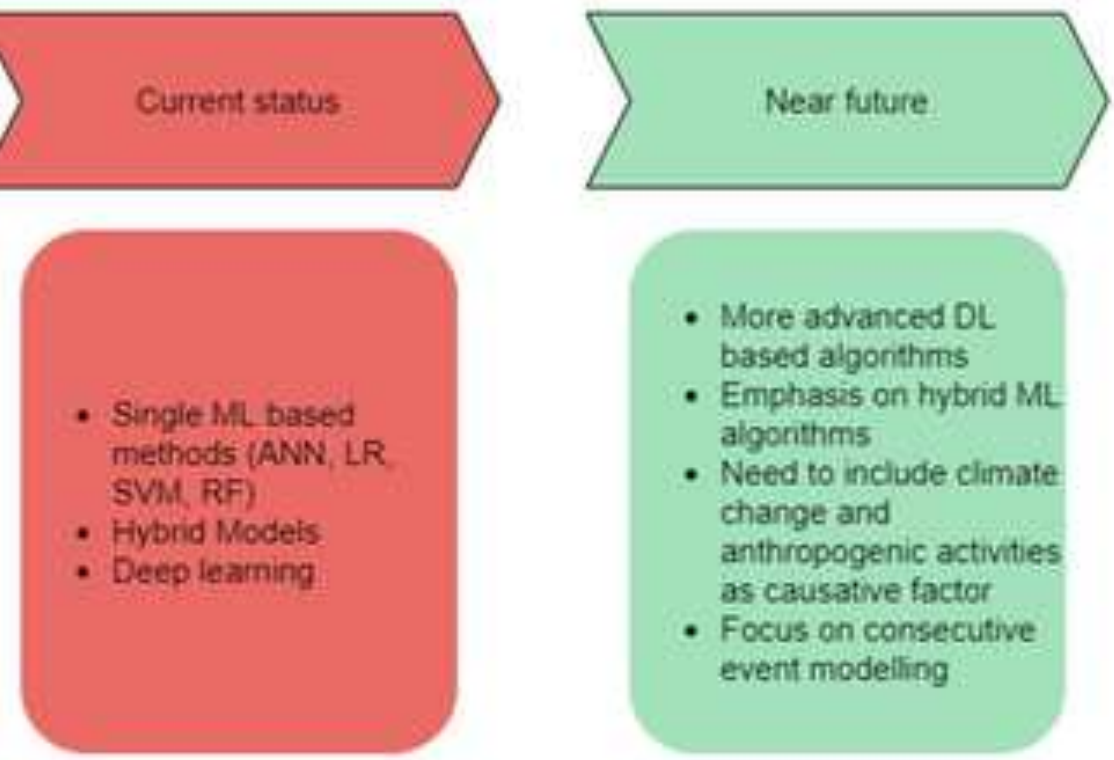

Landslide and debris flow susceptibility

Earthquake

Flood susceptibility

Drought risk

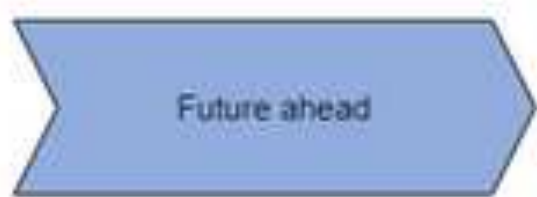

- Decision making abilities

- Predictive capability for up to one week

- Data-diven modeis coupled with physical models for better interpretability of the hazards

- Models with explainable logic and not just black-box 
Click here to download high resolution image

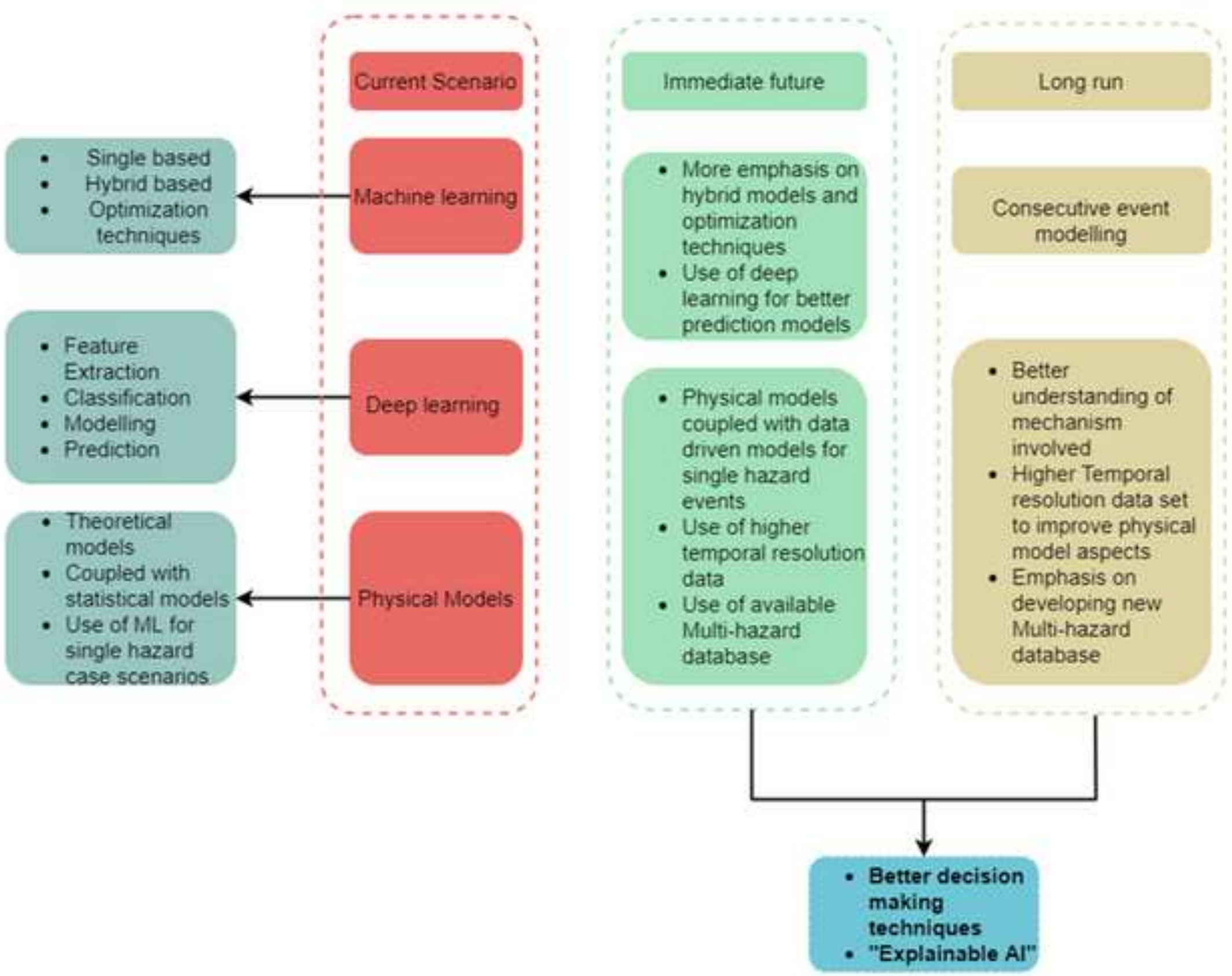


Table 1: Strengths and limitations of various deep learning methods in various geohazard studies.

\begin{tabular}{|c|c|c|c|}
\hline Models & Scientific work & Strengths & Limitations \\
\hline $\begin{array}{l}\text { Convolutional } \\
\text { Neural } \quad \text { Networks } \\
\text { (CNN) }\end{array}$ & $\begin{array}{l}\text { - Susceptibility } \\
\text { (floods, landslides) } \\
\text { (landslide, glacier, flood) } \\
\text { Detection } \\
\text { the captured features of } \\
\text { various geohazards } \\
\text { - Damage } \\
\text { assessment }\end{array}$ & $\begin{array}{l}\text { - Can learn high } \\
\text { level features } \\
\text { - Capture local } \\
\text { geometric features, } \\
\text { spatial patterns }\end{array}$ & $\begin{array}{l}\text { - Could prove } \\
\text { to be difficult to } \\
\text { apply when dealing } \\
\text { with wide diverse } \\
\text { classes of images } \\
\text { - Can overfit } \\
\text { the data }\end{array}$ \\
\hline $\begin{array}{l}\text { Recurrent } \text { Neural } \\
\text { Networks (RNN), } \\
\text { Long Short-Term } \\
\text { Memory (LSTM) }\end{array}$ & $\begin{array}{l}\text { • Temporal evolution } \\
\text { of geohazards } \\
\text { - } \quad \text { Flood forecasting } \\
\text { - } \quad \text { Earthquake trend } \\
\text { prediction and detection } \\
\text { infrastructure damage }\end{array}$ & $\begin{array}{l}\text { - Captures } \\
\text { temporal dynamics } \\
\text { - Additional } \\
\text { connections apart from } \\
\text { direct connections }\end{array}$ & $\begin{array}{l}\text { - Vanishing } \\
\text { gradient and short- } \\
\text { term dependency } \\
\text { - Fails to } \\
\text { represent for short } \\
\text { time, rapidly } \\
\text { changing and non- } \\
\text { periodical data }\end{array}$ \\
\hline $\begin{array}{l}\text { Deep } \\
\text { Networks }\end{array}$ & $\begin{array}{l}\text { - } \text { Landslide } \\
\text { displacement } \\
\text { - Drought } \\
\text { prediction } \\
\text { - Shdex } \\
\text { prediction }\end{array}$ & $\begin{array}{l}- \text { Reduces } \\
\text { parameter tuning } \\
-\quad \text { Efficient } \\
\text { unsupervised training } \\
\text { approach } \\
\bullet \quad \text { More efficient } \\
\text { than single hidden layer }\end{array}$ & $\begin{array}{l}\text { - Visualization } \\
\text { requires } \quad \text { extra } \\
\text { information } \\
\text { processing }\end{array}$ \\
\hline Auto-Encoder & $\begin{array}{lc}\bullet & \text { Feature extraction } \\
\bullet & \text { Landslide } \\
\text { forecasting combined with } \\
\text { LSTM } \\
\bullet \quad \text { Landslide } \\
\text { susceptibility prediction } \\
\bullet \quad \text { Flood detection } \\
\end{array}$ & $\begin{array}{l}\text { - Noise } \\
\text { cancellation } \\
\text { images }\end{array}$ & $\begin{array}{l}\text { Proves to be } \\
\text { efficient only when } \\
\text { the reconstructing } \\
\text { images are similar to } \\
\text { training images. }\end{array}$ \\
\hline
\end{tabular}




\section{Declaration of interests}

$\bigotimes$ The authors declare that they have no known competing financial interests or personal relationships that could have appeared to influence the work reported in this paper.

$\square$ The authors declare the following financial interests/personal relationships which may be considered as potential competing interests:

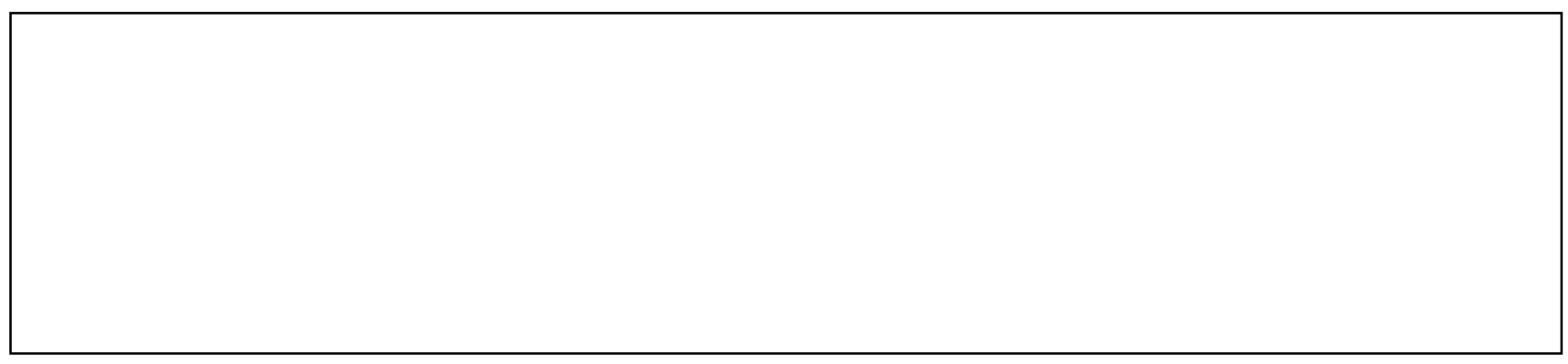


Credit Author Statement: "Conceptualization: A.D., B.P. and M.S.; Methodology: A.D.; Software: B.P.; Validation: A.D. and B.P.; Formal analysis: A.D.; Investigation: A.D. and B.P.; Resources: B.P.; Writing-original draft preparation: A.D.; Writing-review and editing: B.P. and M.S.; Visualization: A.D. and B.P.; Supervision: B.P.; Project administration: B.P.; Funding acquisition: B.P. and A.A." 